\title{
Metamorphosis of a Hairpin Vortex into a Young Turbulent Spot
}

\author{
Bart A. Singer \\ Research Scientist, High Technology Corp. \\ Mail Stop 156, NASA Langley Research Center \\ Hampton, VA 23681-0001 \\ and \\ Ronald D. Joslin \\ Research Scientist \\ Mail Stop 156, NASA Langley Research Center \\ Hampton, VA 23681-0001 \\ March 17, 1995
}

\begin{abstract}
Direct numerical simulation was used to study the formation and growth of a hairpin vortex in a flat-plate boundary layer and its later development into a young turbulent spot. Fluid injection through a slit in the wall triggered the initial vortex. The legs of the vortex were stretched into a hairpin shape as it traveled downstream. Multiple hairpin vortex heads developed between the stretched legs. New vortices formed beneath the streamwise-elongated vortex legs. The continued development of additional vortices resulted in the formation of a traveling region of highly disturbed flow with an arrowhead shape similar to that of a turbulent spot.
\end{abstract}




\section{Introduction}

Hairpin and horseshoe vortices have been proposed as primary structures in turbulent and transitional boundary layers since Theodorsen [1]. In wind-

tunnel experiments, Head and Bandyopadhyay [2] observed hairpin and horseshoe vortices in high Reynolds number, turbulent boundary layers. In flowvisualization studies of turbulent spots, Perry, Lim, and Teh [3] found turbulent spots to be composed of arrays of $\Lambda$-shaped vortices. More recently, Sandham and Kleiser [4] described the development and breakup of hairpin vortices in direct numerical simulations of transitional channel flow. They found that the breakup of the hairpin vortices led to the formation and roll up of new shear layers near the wall and to the subsequent appearance of "wall turbulence."

Studies by Robinson, Kline, and Spalart [5] and Robinson [6, 7] suggest that nearly symmetrical hairpin vortices occur less frequently than asymmetric, or one-sided, hairpin vortices, particularly in fully turbulent flows. However, the pertinent mechanisms are similar, so the continued study of hairpin vortices (the canonical boundary-layer vortex) is justified for both transitional and fully turbulent flows. (See [8] for extensive references on this topic.)

Asai and Nishioka [9] experimentally examined transition at Reynolds numbers below the linear-stability critical point. They excited the flow with strong acoustic forcing near the leading edge of a flat plate. Smoke visual- 
ization of the generated structures resembled that of hairpin vortices. They determined that hairpin vortices, which evolved from the local shear layers, were the key structures that lead to transition.

Peridier, Smith, and Walker $[10,11]$ studied the flow induced by a twodimensional vortex moving parallel to a flat plate in an otherwise stagnant fluid. An unsteady boundary layer develops between the vortex and the surface. The vortex subjects a portion of this boundary layer to an adverse pressure gradient, which leads to the development of a recirculation zone in the viscous region near the surface. Lagrangian coordinates were used to compute the narrowly focused streamwise compression and the wall-normal eruption of fluid particles in the vicinity of the boundary-layer separation. The results are consistent with the theoretical work on separation by Van Dommelen and Cowley [12] and summarized by Cowley, Van Dommelen, and Lam [13]. The theory shows that the separation of near wall fluid starts in a region of locally adverse pressure gradient. Fluid particles are compressed in the streamwise direction and eventually erupt outward from the wall in a violent process. The large but finite Reynolds number calculations of Peridier, Smith, and Walker [11] show that this process can result in the formation of one or more secondary vortices on either side of the local eruption of fluid particles. Smith, Walker, Haidari, and Sobrun [14] cite this eruptive process as one of the most important mechanisms in the reproduction of near-wall vortices in turbulent boundary layers. Their view is based on both the theo- 
retical considerations discussed above and on a series of carefully performed "kernel" experiments.

Experimental work at Lehigh University looked at the development of hairpin vortices generated via different mechanisms. Acarlar and Smith $[15,16]$ considered vortices that were formed behind a hemispherical protuberance and by continuous injection of fluid into the boundary layer. Lowspeed streaklike structures were observed between the legs of the hairpin vortices. They observed that secondary vortices, which appear upstream of the primary vortices, interact with the primary hairpin vortices to form chaotic structures that move rapidly away from the wall. Perhaps because of the low Reynolds numbers used in these experiments, Acarlar and Smith [15, 16] did not observe extensive spanwise spreading of the disturbances. The formation of hairpin vortices that were generated by a pulse of fluid injected into the flow through a streamwise-oriented slit in the wall was considered by Haidari, Taylor, and Smith [17]; Haidari [18]; and Haidari and Smith [19]. Depending upon the Reynolds number and the magnitude and duration of the injection, either single or multiple hairpin vortices formed over the slit. Although Haidari [18] and Haidari and Smith [19] characterized the range of parameters that produced each of the two scenarios, they focused their study on the evolution of single hairpin vortices. As in earlier works, secondary vortices formed upstream and in line with the primary vortex. However, the recent experiments $[17,18,19]$ cited more extensive spanwise 
spreading of the vortex structures than was seen in the earlier Acarlar and Smith [15, 16] experiments. Haidari and Smith [19] distinguished two types of laterally displaced vortices, called secondary and subsidiary vortices. The laterally displaced secondary vortices have vortex heads underneath the legs of the original vortex. Both the in-line and laterally displaced secondary vortices are believed to be initiated by the localized eruption of fluid in an adverse pressure gradient region, as described by Van Dommelen and Cowley [12]. Subsidiary vortices have their heads further displaced from the centerline. These vortices are attributed to "the inviscid deformation of the collective vortex lines comprising the primary vortex." Haidari and Smith [19] speculate that the repeated formation of secondary and subsidiary vortices ultimately results in a turbulent spot.

Other experimental studies that focused explicitly on early turbulent-spot development were performed by Amini and Lespinard [20]. They excited a disturbance in the boundary layer with a loudspeaker mounted beneath a small hole in the wall of a flat plate. The estimated vertical velocity created by the loudspeaker was as large as 1.25 times the free-stream velocity. The perturbation velocities on the centerline directly downstream of the hole were negative, but further downstream the flow along the centerline was characterized by a relative velocity surplus with regions of velocity deficit that straddled the velocity surplus region. A similar bridging of other velocity surplus regions located further from the centerline occurred. The data 
strongly suggest the existence of several strong hairpin vortices in the flow before the turbulent-spot stage is reached.

Results from the direct numerical simulation of a hairpin vortex in a timedeveloping boundary layer were reported by Singer [21]. His calculations showed that a secondary vortex head appeared upstream of the primary vortex. Stability calculations suggested that a rapid instability mechanism might be responsible for the roll up of the secondary vortex head. The calculation was discontinued when the streamwise periodicity assumption of the flow began to interfere with the results.

The computational studies of Henningson, Lundbladh, and Johansson [22] have helped to clarify the connection between the later stages of transition and the early stages of turbulence. In their direct numerical simulations of the early stages of turbulent-spot formation in both channel and boundary-layer flows, they used initial conditions that consisted of pairs of counterrotating vortices oriented at various angles to the flow direction. The growth of the kinetic energy of the localized disturbances was strongly dominated by the part of the disturbance associated with the wall-normal vorticity that was generated by a three-dimensional lift-up effect. The low streamwise wave-number disturbances grew algebraically for long periods of time, which facilitated the formation of elongated structures in the streamwise direction. They demonstrated that a transient linear mechanism was responsible for the growth of these structures. The larger structures interacted nonlinearly 
to put energy into increasingly small spanwise scales. This process creates the long streaky structures that are typical in turbulent flows, regardless of the initial conditions.

The results presented here are another step toward understanding the relationship between localized disturbances and the onset of turbulence. In section 2, we discuss the numerical details, including the disturbance initiation and the resolution requirements for the calculation. The results are discussed in section 3, and our conclusions are presented in section 4 .

\section{Numerical Model}

\subsection{The Computer Code}

We used a variation of the spatially developing direct numerical simulation code described in Joslin, Streett, and Chang [23, 24] to solve the incompressible Navier-Stokes equations for the flow over a flat plate with a zero pressure gradient. In the spatially developing code, disturbances develop as they would in a physical experiment. A buffer-domain technique, which is described in Refs. [23, 24, 25], provides nonreflective boundary conditions in the streamwise direction by slowly parabolizing the equations in the buffer region. The results are only valid (and of course only presented) for the flow in the physical domain. The streamwise discretization employs sixthorder compact differences for the calculation of the velocity derivatives and fourth-order compact differences for the calculation of the pressure gradi- 
ents. A hyperbolic-tangent stretching previously used by Zang and Hussaini [26] concentrates the streamwise grid points in the most active region. The computational domain is periodic in the spanwise direction and is symmetric about the centerline; hence, Fourier cosine and sine expansions accurately represent the variations in this direction. In the wall-normal direction, perturbations are expanded in terms of Chebyshev polynomials on a grid with a clustering of grid points in the boundary layer. A staggered mesh permits the calculation of the pressure at the Gauss points, rather than the Gauss-Lobatto points that are used for the calculation of the velocity. The solution is advanced in time with a time-splitting procedure that employs an implicit Crank-Nicolson scheme for the wall-normal diffusion terms and an explicit three-stage Runge-Kutta scheme for all remaining terms. The influence-matrix technique is used to solve for the two-dimensional component of the pressure while the the three-dimensional components of the pressure are solved directly. Additional details of the code and its validation are given in references [23] and [24].

\section{$2.2 \quad$ Initialization}

A plan view of the geometry of the problem is illustrated in Fig. 1. The streamwise direction is denoted by $x$; the wall-normal direction, by $y$; and the spanwise direction, by $z$. The corresponding perturbation velocities are $u, v$, and $w$. The total velocity in the streamwise direction $U$ is the sum of the perturbation velocity and the local Blasius profile $U_{b}(x, y)$. All quanti- 
ties are nondimensionalized with respect to the free-stream velocity $U_{e}$ and a reference length $\delta_{0}^{*}$. The reference length is equal to the displacement thickness $\delta^{*}$ at the point where the Reynolds number (based on the displacement thickness) equals 530 (i.e., $R e_{\delta_{0}^{*}}=U_{e} \delta_{0}^{*} / \nu=530$, where $\nu$ is the kinematic viscosity). Streamwise distances are referenced to this point. Time $t$ is nondimensionalized with $\delta_{0}^{*} / U_{e}$. Although a calculation at a higher Reynolds number might be desirable, spatial resolution requirements constrain the choice of Reynolds number for this calculation. From $x=50$ (where the local displacement-thickness Reynolds number is approximately 600 ) to $x=75$, fluid is injected into the flow at a uniform rate with a vertical velocity on the centerline equal to $v_{0}(t)$. Over a length $2 \delta_{0}^{*}$ upstream and downstream of the slot, the vertical velocity decreases exponentially with a decay rate of $2 \log 2$. In the spanwise direction, the injection velocity decreases linearly from its value on the centerline $(z=0)$ to 0 at $z= \pm 1$. The flux through the slot is the same as that for a slot that is $1 \delta_{0}^{*}$ wide and $26.35 \delta_{0}^{*}$ long with a uniform vertical velocity of $v_{0}(t)$. The nominal injection velocity $v_{0}(t)$ is constant at 25 percent of $U_{e}$ for time $0.5 \leq t \leq 4.5$. This injection velocity is linearly ramped to this constant value for times less than 0.5 and is linearly ramped to 0 by $t=5$. Although the parameters chosen for this numerical experiment do not precisely match a specific physical experiment, these parameters correspond approximately to the highest Reynolds number water-channel experiments of Haidari [18]. However, in the numerical 
simulation, the injection velocity $v_{0}$ is greater than that in the water-channel experiments for such a large Reynolds number. The larger initial disturbance helps to ensure that events that are characteristic of the late stages of transition will occur within the time frame of the calculation. No free-stream turbulence or inflow seeding of the disturbances is used.

\subsection{Resolution}

The present results were obtained from a simulation in which the initial grid resolution employed 301 streamwise grid points, 121 wall-normal grid points, and 41 spanwise grid points $(301 \times 121 \times 41)$. The energy spectrum of the Chebyshev and Fourier coefficients was routinely monitored. Extensive gridresolution tests were performed periodically. The code was run several times with additional grid points for a time window approximately $1 \delta_{0}^{*} / U_{e}$ wide. Local velocity changes of more than 1 percent were unacceptable; the grid resolution was adjusted until the variation between runs was within the 1 percent limit. Streamwise and wall-normal grid resolutions were adjusted by varying the grid stretching, the domain length, and the number of grid points. The resolution used for the bulk of the calculation $(21.0 \leq t \leq 91.5)$ employed a $261 \times 161 \times 121$ grid with a streamwise length of $60 \delta_{0}^{*}$, a wallnormal height of $40 \delta_{0}^{*}$, and a spanwise half-width of $10 \delta_{0}^{*}$. With this grid, the Chebyshev spectrum of the perturbation velocity components in the vertical direction indicated a decay of $10^{6}$ in the square of the expansion coefficients from the low to high wave numbers. The Fourier spectrum in the spanwise 
direction indicated a decay of $10^{7}$ for a corresponding quantity. For the time $91.5<t<145.9,183$ points were used in the vertical direction to maintain the $10^{6}$ decay in the square of the Chebyshev coefficients. For $101.8<t<145.9$, the decay in the square of the Fourier coefficients was $10^{4}$, which would suggest that the calculation had spatial discretization errors in the velocity and pressure fields of approximately 1 percent. (The vorticity would be expected to exhibit larger errors than the velocity field.) These spatial errors were difficult to discern on plots of the data; repeated runs at different resolutions showed variations less than 1 percent in velocity and pressure. (See below for more details.) At $t=139.5$, the extent of the streamwise domain was increased to $90 \delta_{0}^{*}$, and the number of streamwise grid points was increased to 351. This increase was needed to maintain the growing patch of disturbances in the computational domain. At $t=145.9$, the number of vertical grid points was reduced to 161 , and the number of points in the spanwise direction was increased to 161 , which utilized all of the available computer memory. Until $t=158.3$, the decay of the square of the Chebyshev coefficients was $10^{5}$; that of the Fourier coefficients was $10^{4}$. Velocity and pressure field discretization errors of more than 1 were likely in the data obtained for $t>158.3$; therefore these results are not presented. If a larger Reynolds number were used, the spatial resolution would become inadequate at an earlier time.

At $t=116.7$, the results suggested that the spanwise growth of the struc- 
ture was inhibited by the limited length of the spanwise domain. To check this possibility, the flow field at $t=101.8$ was interpolated onto a grid with twice the spanwise wavelength but with the same number of grid points. The perturbation velocities at the edge of the original domain were damped to 0 to ensure a smooth transition to the larger domain. The calculation with the expanded domain had a Fourier power spectrum with a decay of $10^{4}$, rather than $10^{7}$. However, the computed flow fields during the time of overlap between the calculations $(101.8 \leq t \leq 116.7)$ were difficult to distinguish visually, which suggests that neither the narrow domain nor the marginal resolution had a significant impact on the major flow features. The remainder of the calculation was performed on the wider domain.

\section{Results and Discussion}

\subsection{Early developments}

The injected fluid creates a large initial disturbance that both creates new vorticity in the flow and redistributes the existing mean-flow vorticity. The new vorticity comes from two sources, the most obvious of which is the velocity distribution of the injected fluid. The spanwise distribution of vertical velocity in the pulse introduces streamwise vorticity directly into the flow. At the upstream and downstream ends of the slit, spanwise vorticity is introduced as a result of the streamwise variation of the vertical velocity. Another source of vorticity is the boundary vorticity flux associated with 
the wall pressure gradient, which is created by the original disturbance [27]. Because the injected fluid initially has no streamwise momentum, a streamwise velocity deficit is created. Even after the injection is discontinued at $t=5.0$, the mass of slow-moving fluid blocks the fluid from upstream. The fluid decelerates as it approaches the slit, and a high-pressure region forms at the upstream end of the slit, which imparts some momentum to the injected fluid. The approaching fluid is deflected over and around the injected fluid. The streamwise vorticity in the injected fluid diffuses outward, which draws the surrounding fluid near the wall toward the centerline. Figure 2 illustrates some of these important features at $t=15.0$. The velocity vectors on the left-hand side of the figure indicate the centerline velocity upstream of the injection slot. The injection slot is illustrated as a dark horizontal line under the structures. In the figure, a streamline that starts shortly upstream of the injection slot and slightly off of the centerline deflects both wallward and away from the centerline as it passes through the high-pressure region. The same streamline enters the low-pressure zone and moves both upward and toward the centerline; it eventually spirals around the elongated low-pressure tube. A weak vortex head rises at the downstream end of the low-pressure region, and a larger mass of low-pressure fluid collects at the upstream end. The minimum pressure and the most intense swirling motions are associated with the upstream low-pressure region. The weaker vortex at the downstream end of the slot diffuses and does not appear to play a role in the subsequent 
developments.

The proximity and shape of the high-pressure zone strongly influences the fluid that enters the low-pressure region. Near-wall fluid that approaches the high-pressure region from upstream is deflected wallward and laterally in a manner similar to the streamline motion shown in Fig. 2. A small highpressure region develops in the stagnation area as the deflected fluid encounters the wall. This new high-pressure region further deflects the approaching fluid. The important features are illustrated in Fig. 3 at $t=21.75$, where the original and secondary high-pressure zones are indicated. This figure shows grey-scale contours and superimposed contour lines of the pressure in a $y-z$ plane at $x=55.1$. The light shading indicates areas with high pressure, the darker shading indicates areas with low pressure. Three streamlines, which are restricted to the plane $x=55.1$, are shown; these are not true streamlines but are the integral curves of the two-dimensional field $(v, w)$ in the plane $x=55.1$. The lowermost line shows that as fluid particles approach the side of the secondary high-pressure region they form a strong vortex with positive streamwise vorticity. Downstream of the high-pressure regions, this vortex enters the large low-pressure region seen in Fig. 2 and coalesces with the positive vorticity created by the upward motion of the injected fluid. The strongest swirling motions in the flow are associated with this vortex interaction. Fluid particles that approach the secondary high-pressure region with very obtuse angles are pushed away from the wall. (See the middle line in 
Fig. 3.) Large deflections can be experienced by some fluid particles, as seen in the uppermost line in Fig. 3. This deflection is the start of a weak vortex with a negative streamwise vorticity. Greco [28] and Smith, Walker, Haidari, and Sobrun [14] refer to three-dimensional vortices that form in the end-wall boundary layer upstream of an obstacle as "necklace" vortices. Later, we will see that the weak vortex with negative streamwise vorticity has many of the same features as a necklace vortex. In this flow, the injected fluid acts as a slow-moving obstacle.

As the flow develops, the most vigorous disturbances develop from the upstream low-pressure region. By comparison, the vortex head, which rises at the downstream end of the slit, has a minimal effect on the subsequent disturbances. This difference in development is at least partially due to the presence of the high-pressure region, which increases the amplitude of the swirling flow at the upstream end of the slit. The highly unstable velocity profile over the slit itself is another possible cause for the rapid development of the upstream disturbance. Except for the upstream and downstream ends of the injection slot, the streamwise velocity profiles over the injection slot are quite similar and have at least one inflection point. Figure 4 shows a typical streamwise velocity profile over the slit region on the centerline at $t=15$. The Blasius profile is included for comparison. The inviscidly unstable velocity profile can lead to very rapid growth of the disturbance.

All of the structures convect downstream. The downstream low-pressure 
region is passive and becomes relatively unimportant. As more fluid from upstream is blocked by the slow-moving injected fluid, the intensity of the high-pressure region increases, and it maintains its relative position just upstream of the low-pressure zone. The upstream low-pressure region becomes stronger as it forms into a well-defined hairpin vortex as shown in Fig. 5. A plan view of the structure in Fig. 6 at $t=42.0$ indicates that the vortex has a rounded head, which connects two legs that approach each other and then separate further upstream. This shape is the canonical form of hairpin vortices, which are so named because they so nearly resemble the metal clips used to hold hair in place. Here we use the term "hairpin" to refer to a vortical structure with a spanwise head that connects the approximately streamwise legs, which trail upstream. In Fig. 5, two dramatic changes occur in the slope of the low-pressure region. The near-wall structure makes an angle of about $30^{\circ}$ with the wall. At a height $y \approx 2$, the structure is approximately parallel to the wall. Further downstream, the vortex head gradually increases its slope toward the edge of the boundary layer. By examining the flow inside of the vortex core, a significant vertical velocity can be seen that leads away from the wall inside the low-pressure region. Helicity (the dot product of velocity and vorticity) has a large amplitude in the low-pressure region, except for the hairpin vortex head. In the head, the vorticity is primarily spanwise; the lack of any significant spanwise velocity in the head region results in nearly no helicity. On the centerline, the symmetry con- 
straint results in exactly zero helicity. The velocity field inside the vortex can be represented by streamlines. Because the flow is unsteady, the streamlines do not represent actual particle paths. Here, we calculate streamlines in the laboratory frame of reference with starting locations near the foot of the hairpin vortex. The streamlines remain inside the low-pressure region until $y \approx 2$; then the streamlines exit the vortex and travel downstream. The pumping of fluid away from the wall inside a hairpin vortex has been observed experimentally by Hagen and Kurosaka [29]. Streamlines initiated in the vortex head and integrated both forward and backward show a jump in vertical displacement toward the wall in the vicinity of the vortex head. The large vertical velocities at the upstream and downstream ends of the vortex head are responsible for this jump.

Just as streamlines are useful in understanding the velocity field, vorticity lines (also known as vortex lines) are used to understand the vorticity field that is associated with the vortex. Unlike streamlines, vorticity lines are independent of frame of reference. A number of vorticity lines are computed with initial locations inside of the vortex head. The lines remain inside the low-pressure region for varying distances, then eventually exit and continue in the spanwise direction. A few of the lines remain inside the low-pressure region until the foot of the vortex is reached. A lack of strong cohesiveness of the vorticity lines, particularly in the lower portions of the boundary layer, is typical. The rapid increase in spanwise vorticity as the wall is approached 
causes the vorticity lines that emerge from the low-pressure zone to move primarily in the spanwise direction, although some of the lines have a weak streamwise kink similar to that illustrated in Fig. 6(b) of Smith et al. [14]. However, unlike the results reported in Ref. [14], the kink in the vorticity line is not dynamically significant in this case. The calculations in Ref. [14] that suggest the importance of the kinks in the vorticity lines and the development of these kinks into subsidiary vortices are essentially inviscid. As a result, these kinks may have greater significance at higher Reynolds numbers than that which is used here.

\subsection{Auxiliary flow structures}

In addition to the primary hairpin vortex, various other flow structures develop. Although the origin of the structures discussed in this section may be linked with the initial conditions, these structures first become significant long after the initial injection has ceased.

A contour plot of pressure surfaces on a plane $y=0.53$ at $t=42.0$ is illustrated in Fig. 6. The hairpin vortex is indicated by the light grey surface. The downstream end of the injection slot is outlined by the rectangle. A region of high pressure exists between the feet of the vortex. A U-shaped area of low pressure is upstream of the high-pressure region. Velocity vectors in this region reveal that the core of the vortex with the negative streamwise vorticity observed in Fig. 3 is located in this low-pressure region. Earlier, we suggested that this vortex is a moving necklace vortex created by the 
obstruction of the injected fluid. The horseshoe shape of the vortex, which is closed at the upstream end and open at the downstream end with the highpressure stagnation region inside, confirms that this is indeed a necklace vortex.

The low-pressure concentration between the vortex legs portends the appearance of secondary vortices in this region. By $t=50.25$, a secondary hairpin vortex head has developed upstream of the primary vortex head. A side view of the high- and low-pressure surfaces at $t=50.25$ is shown in Fig. 7. The extensive elongation of the vortex is clearly visible. Midway through the elongated region between the head of the primary hairpin vortex and the "knee," where the vortex slopes sharply towards the wall, a second vortex head appears. Selected vorticity lines initiated in each of the two vortex heads extend through the low-pressure region to exit near the foot of the vortex. The vorticity lines then move primarily in the spanwise direction with small streamwise kinks similar to those discussed above. Vorticity lines that go through the two distinct vortex heads do not intertwine in the common low-pressure region. The almost circular cross section of the low-pressure region that marks the legs of the vortices and the lack of intertwining of the vorticity lines suggests that the primary and secondary vortices merge rather than retain individual identities. In a higher Reynolds number turbulent boundary layer, the situation could be different.

The appearance of multiple vortex heads in a hairpin vortex structure 
is not unusual; multiple heads have been observed in previous experiments $[15,16,17,18,19]$ and calculations [21]. With regard to a similar computation in a temporally developing boundary layer, Singer [21] suggested that the secondary vortex head appears as a result of an inviscid instability in the flow region between the vortex legs. He solved the two-dimensional OrrSommerfeld equations with a mean flow equal to the velocity profile over the slit and found unstable waves that grow hundreds of times faster than the most unstable wave for the Blasius profile at that Reynolds number. Although the same procedure could be applied here, (Fig. 4 illustrates a highly unstable shear layer that exists over an extensive streamwise distance) in this flow the unstable-wave argument raises more questions than it resolves. In the previous work [21], the fluid injection was through a square hole for a much longer duration. The primary hairpin vortex formed at the upstream end of the hole and was convected over and downstream of the injection hole while fuid was still being injected. In that case, the unstable velocity profile that was used in the Orr-Sommerfeld calculation existed largely upstream of the primary hairpin vortex head. In the current calculation, fluid was injected through a long, thin slit for a comparatively short time. The unstable velocity profile shown in Fig. 4 exists upstream and downstream of the primary vortex head, but a secondary vortex head appears only upstream of the primary head. Hence, the existence of an unstable velocity profile might be a helpful or perhaps even necessary condition for the development of the secondary 
vortex; however, by itself, it is not sufficient.

Smith et al. [14] and Haidari and Smith [19] hypothesize that secondary vortex formation follows an ejection of fluid away from the wall, as explained by the theory of Van Dommelen and Cowley [12]. In the theory, local transient adverse pressure gradients must act on a region of near-zero vorticity fluid. This mechanism appears to operate here. Figure 8 illustrates the pressure contours on the flow centerline at times $t=26.5, t=35.7$, and $t=42.0$. The high-pressure region located downstream and below the primary hairpin vortex head creates an adverse pressure gradient for the fluid between the hairpin vortex legs. Note that in this case the adverse pressure gradient need not extend all the way to the wall. The initial injection has already provided a large reservoir of near-zero-vorticity low-momentum fluid away from the wall. The secondary vortex head forms on the upstream side of an additional upwelling of this fluid. Although this process is not as dramatic as the eruption of near-wall fluid seen in the Lagrangian calculations of Peridier, Smith, and Walker [11] for high Reynolds number flows, the basic elements of the processes seem to be the same even for this low Reynolds number.

The flow at $t=50.25$ contains at least three distinct vortices. In addition to the primary and secondary vortices head, another vortex can be educed. In Fig. 7, the low-pressure regions below the elongated section of the hairpin vortex are actually part of the new vortex. Evidence can be seen more clearly in Fig. 9, which provides a view of the low-pressure region from upstream. 
Three streamlines that are restricted to the plane $x=71.4$ (the location of the vertical line in Fig. 7), are shown in the figure. Arrows indicate the direction of the flow. The swirling motion associated with the new low-pressure region is opposite to that of the hairpin vortex legs. At the same distance from the wall but further from the centerline, another region of weakly rotating flow can be seen. These two regions of swirling flow merge with each other further downstream to form an asymmetric U-shaped vortex, which is closed at the downstream end. The vorticity is more concentrated in the leg near the symmetry plane and more diffuse in the outboard leg.

The location of the new U-shaped vortex (relative to the primary hairpin vortex) is similar to that of the laterally displaced secondary vortices observed by Haidari and Smith [19]. However, in this case, a portion of the U-shaped vortex seems to be closely connected to the necklace vortex that was created by the blockage due to the injected fluid. This connection can be more clearly seen if the outboard leg of the U-shaped vortex is followed upstream. In Fig. 10 , cross-stream velocity vectors at $x=67.3$ are shown superimposed on greyscale contours of the pressure in the plane. The dark shading represents lowpressure areas and the light shading represents high-pressure areas. Three regions of rotating flow can be distinguished. The leg of the hairpin vortex on the left side of the figure has a core of low pressure. The counterclockwiserotating necklace vortex that is higher in the boundary layer and to the right of the hairpin vortex is also associated with low pressure. A weak, 
clockwise-rotating system near the wall and further to the right is the diffuse beginning of the U-shaped vortex. The hairpin and necklace vortex legs induce a spanwise flow near the wall in the direction of increasing $|z|$. The rise of near-wall fluid just to the left of the U-shaped vortex is uncorrelated with either a spanwise or streamwise adverse pressure gradient at this and earlier times in the simulation. The movement of this fluid away from the wall is partially a viscous entrainment effect of the necklace vortex. A mild rebound of the fluid that is pushed toward the wall between the hairpin and necklace vortices may also account for some of the vertical motion. The unsteady boundary-layer separation theory of Van Dommelen and Cowley [12] does not apply in this case.

Further downstream, the primary hairpin vortex head decays, but other structures in the flow strengthen. Figure 11 shows a plan view of high- and low-pressure surfaces. The original hairpin vortex head does not appear at the pressure level used for this figure. The low-pressure region that connects the secondary vortex head and the vortex legs also does not appear in the figure. The high-pressure region at the upstream end of the structures is greatly reduced in size. However, in addition to a tertiary vortex head that forms between the original hairpin vortex legs, the U-shaped vortex that was suggested in Figs. 7, 9, and 10 appears as a dominant structure (at least in size). The counterclockwise-rotating necklace vortex seen in Fig. 10 (Fig. 3 shows this necklace vortex beginning to form) appears as the low-pressure 
regions on the top and bottom of the figure.

\subsection{Vortex regeneration}

In time, the large U-shaped vortex under the hairpin vortex legs loses some of its coherence, the tertiary hairpin vortex head strengthens, and new vortices develop. Top and side views at $t=97.2$ are shown in Figs. 12 and 13, respectively. The level for the high-pressure surface is $p=0.02$, which is less than that used in previous figures. The original high-pressure zone is coherent; however, it is not as strong as before. A new vortical structure has formed under the remnants of the large U-shaped vortex. Analysis shows that this new structure consists mostly of streamwise vorticity, and it is aligned primarily in the streamwise direction. In the low-pressure core shown in the figures, the streamwise vorticity is between $-1.0 U_{0} / \delta_{0}^{*}$ and $-1.5 U_{0} / \delta_{0}^{*}$. Some vertical vorticity $\left(\approx 0.5 U_{0} / \delta_{0}^{*}\right)$ exists in this region. In their studies of low Reynolds number, turbulent flows, Robinson, Kline, and Spalart [5] (see also [7] and [8]) observed a multitude of similar structures and labeled them "quasi-streamwise vortices." We will retain the same nomenclature here. In Fig. 12, the high-pressure surface located near the new quasi-streamwise vortex actually separates this vortex from another similar quasi-streamwise vortex that is slightly upstream and weaker (so it does not appear as a low-

pressure surface). The high pressure develops where the cross-stream velocity vectors of the two corotating vortices stagnate.

The continued development of strong vortices suggests that the distur- 
bances are regenerative and have only a weak relationship to the originally input disturbance. Although each individual vortex forms in its own particular environment, some processes are more commonly observed than others.

One commonly observed situation is illustrated in Fig. 14, which represents the activity on a cross-sectional plane at $x=98.7$ and $t=97.2$. This plane cuts through the middle of the remnant of the U-shaped vortex seen in Figs. 12 and 13. Dark areas in the figure are low-pressure areas; light areas are high-pressure areas. The grey surface on the right represents the portion of the U-shaped vortex that protrudes through the plane. Vector lines in the figure indicate the velocity in the plane. The lines with arrowheads are streamlines that are restricted to the plane. One long spiraling line on the right indicates the circulating flow associated with the leg of the Ushaped vortex. This vortex induces a flow of near-wall fluid to the left. Three streamlines restricted to the plane show flow from right to left near the wall. This fluid cannot negotiate the adverse pressure gradient. If we move from the dark, low-pressure region to the lighter, higher pressure region, then the restricted streamlines lift away from the wall and concentrate in a relatively narrow band. Near-wall fluid on the left side of the figure is driven from the higher pressure region to the lower pressure region. Two plane-restricted streamlines near the wall go from left to right until they are entrained in the ejection of near-wall fluid. The rising fluid creates a new vortex to the left of the ejection zone. Although this flow is complicated by both the presence of 
other vortices (the other leg of the U-shaped vortex can be faintly discerned from the velocity vectors on the left, the necklace vortex is upstream and to the right of the figure, and the leg of the original hairpin is above this figure on the left) and a mean shear flow that goes into the plane of this figure, the basic ingredients of the Van Dommelen and Cowley [12] unsteady surface separation theory exist. In a manner similar to that observed by Peridier, Walker, and Smith $[10,11]$, a strong vortex moves near-wall fluid into an adverse pressure gradient, the fluid erupts away from the wall, and a new vortex forms in a shear layer created by the eruption.

We found the above unsteady surface separation process to occur frequently in crossflow $(y-z)$ planes, but we found no surface eruptions initiated by streamwise adverse pressure gradients. Although earlier we observed that a streamwise adverse pressure gradient initiated the formation of the secondary vortex shown in Figs. 7, 8, and 9, the low-vorticity fluid that was uplifted came from the original fluid injection. When exposed to a streamwise adverse pressure gradient, the fluid was further uplifted and a secondary vortex head formed. Similar events occurred later in the computation. The low-vorticity fluid that was uplifted originated from injected fluid or fluid that was moved away from the wall by quasi-streamwise vortices. The vortex heads seemed to be too far from the wall to provide the necessary adverse pressure gradient there. In a high Reynolds number boundary layer, the length scales of the vortical structures relative to the boundary-layer thick- 
ness are much smaller; hence, the vortex heads are relatively closer to the wall and might induce the necessary adverse pressure gradient nearer to the wall.

An example of a vortex head that forms from fluid ejected by quasistreamwise vortices is shown in Figs. 15(a) and 15(b). These figures represent the crossflow plane at $x=128.2$ at time $t=148.0$. The crossflow velocity vectors and streamlines restricted to the plane are the same in both figures. However, in Fig. 15(a), the dark regions indicate low pressure, the lighter regions indicate high pressure. In Fig. 15(b), the magnitude of the vorticity is high in the dark regions and low in the light regions. A strong clockwise-rotating vortex dominates the center of the figures. This vortex induces a cross-stream flow from right to left. As near-wall fluid moves from the low-pressure zone on the right to the higher pressure zone left of the clockwise-rotating vortex, the fluid rises rapidly away from the wall. The restricted streamline shown becomes entrained in a new near-wall vortex created in the shear layer of the ejection. In Fig. 15(b), the fluid in the center of the ejection has a relatively low vorticity. The activity in these figures is distinguished from other ejections in the crossflow plane in that a "cap" of high-vorticity fluid exists on top of the counter-rotating streamwise vortices. This cap is mostly spanwise vorticity, and it develops into the head of a laterally displaced hairpin vortex. Although it develops in a region of mildly adverse streamwise pressure gradient, the streamwise adverse pressure 
gradient does not extend into the near-wall region. The streamwise adverse pressure gradient may help determine the location at which the vortex head forms, but the entire ejection process in this case seems to be controlled by quasi-streamwise vortices. The importance of the quasi-streamwise vortices in initiating the regenerative process, particularly near the wall, is consistent with Robinson's [6, 7] observation of a large population of quasi-streamwise vortices in the near-wall region and the existence of transverse vortices farther from the wall.

The multitude of flow structures present at $t=153.10$ are shown in Fig. 16, where a top view of isopressure regions are shown. The hairpin vortex at the upstream end of the figures has features that are almost identical to those of the hairpin vortex in Fig. 5. The laterally displaced secondary hairpin vortices to each side are the same structures that were developing in Figs 15(a) and 15(b). The potato-shaped regions of high pressure can be used to estimate where adverse pressure gradients exist. Several quasi-streamwise vortices in Fig. 16 are numbered in the order of their appearance at this pressure level. The typical quasi-streamwise vortex first appears under the leg of another elongated vortex. Although the effects of the initial injection persist for a long time, the appearance of the quasi-streamwise vortices seems to be in response to local flow conditions in the boundary layer (e.g., conditions similar to those illustrated in Fig. 14 rather than anything directly related to the original injection of fluid), so the mechanics of their development is 
probably generic. The region labeled vortex 1 is part of the quasi-streamwise vortex that formed under the remnants of the U-shaped vortex in Figs. 12 and 13. This quasi-streamwise vortex grows very long, lifts up in the boundary layer, and then slowly decays. The high-pressure region that separates vortex 1 from vortex 2 is shown in Fig. 12. Another high-pressure region separates vortex 2 from vortex 3 , and a small high-pressure region adjacent to vortex 3 suggests that perhaps another quasi-streamwise vortex exists, but is not yet visible at this pressure level. The vortices 1, 2, 3, and 4 all corotate and are dominated by negative streamwise vorticity. Vortex 4 closely resembles the early stages of vortex 1 . The formation rate of all of the new vortices exceeds the rate at which the vortices decay, which leads to the growth of the patch of vortices.

\subsection{Relationship to a turbulent spot}

The collection of individual vortex structures may be part of a nascent turbulent spot. In an experiment, the outline of a turbulent spot is often determined either by a contour of the perturbation streamwise velocity $[30,31,32,33]$ or by some level of the intermittency of the flow $[34,35]$. For a nonstationary flow, the intermittency is usually determined as an ensemble average of a telegraph signal. Because only one realization of the flow was computed, the intermittency itself could not be obtained here. However, the intermittency is a measure associated with rapid changes in the flow, and the direct numerical simulation provides a variety of possible comparable 
measures. Here, the magnitude of the vertical vorticity of the flow is used. This quantity measures the spanwise changes of the streamwise velocity and the streamwise changes of the spanwise velocity. No vertical vorticity exists in the original laminar flow, and the input disturbance contains no vertical vorticity (although vertical vorticity is produced quickly from the tilting of the streamwise vorticity). Figure 17 illustrates plan views of contours of the magnitudes of the perturbation streamwise velocity (top) and the vertical vorticity (bottom) at $t=150.10$ in a plane on which $y=2.41$. A $0.02 U_{0}$ minimum contour level for the perturbation velocity is used, and a $0.02 U_{0} / \delta_{0}^{*}$ minimum contour level is used for the vertical vorticity. Both approaches reveal an arrowhead shape that points downstream with a quiescent zone along the centerline of the upstream end of the spot. A side view of these contours near the centerline is shown in Fig. 18. (On the centerline, the symmetry condition gives zero vertical vorticity.) The leading edges of the enveloping curves are away from the wall, which suggests the start of the overhang region of a turbulent spot. The long tail in the streamwise perturbation velocity at the upstream end of the disturbance is similar to what was observed experimentally by Barrow, Barnes, Ross, and Hayes [33] in their Fig. 4.

Wavelike features near the wing tip (the upstream spanwise edge) of a turbulent spot were first observed by Wygnanski, Haritonidis, and Kaplan [36]. Flow visualization experiments by Chambers and Thomas [37] sug- 
gested that these waves were produced by the turbulent spot, but had very little to do with the subsequent dynamics of the turbulent spot. Further work by Glezer, Katz, and Wygnanski [34] show that the waves sometimes result in the formation of new turbulent regions that amalgamate with the existing turbulent spot. Previous calculations by Henningson, Spalart, and Kim [38] did not show waves in the boundary-layer calculations, but did report waves in the channel-flow calculations. At this stage of our direct numerical simulation, no indication of a wave packet is detected near the turbulent spot wing tip.

Whether the flow inside the young turbulent spot is fully turbulent or only highly intermittent is difficult to determine. In evaluating the results of a temporal direct numerical simulation of a turbulent spot in plane-channel flow, Henningson and Kim [39] looked at locally averaged quantities. The existence of a small, but noticeable, log-law region led them to conclude that in the core of the spot the flow was, in fact, turbulent. A similar zonal calculation has been performed here at $t=158.30$. Velocity profiles for a section of the highly intermittent area with $120 \leq x \leq 132$ and $|z| \leq 3.0$ have been averaged and plotted as $u^{+}$versus $y^{+}$in Fig. 19. The solid line represents the results of the direct numerical simulation; the dotted line represents the expected behavior in the $\log$ layer $\left(u^{+}=1 / \kappa \log y^{+}+C\right.$, where $\kappa=0.4$ and $C=5.0$ ); and the chain-dot line represents the expected behavior in the viscous sublayer $\left(u^{+}=y^{+}\right)$. Although the computed results 
have a small region where $u^{+}$varies like $\log y^{+}$, the profile does not resemble a fully turbulent boundary-layer profile. We believe that most of the typical, turbulent coherent structures are present at this stage of the calculation, but their temporal and spatial scales are not yet representative of a fully turbulent boundary layer. If the calculation were continued and a larger, more mature turbulent spot were allowed to develop, then the averaged profiles would be expected to more closely approximate the fully turbulent profile.

\section{Conclusions}

A direct numerical simulation of the evolution of a localized disturbance first into a hairpin vortex and then into an immature turbulent spot was performed. The resolution of the calculation was sufficiently fine so that the details of the developing flow could be observed more clearly than in previous investigations. A short pulse of fluid, injected through a wall slot, blocked part of the usual boundary-layer flow. The blockage formed a high-pressure region at the upstream end of the slot. A strong hairpin vortex formed just downstream of the high-pressure region. A weak hairpin vortex head emerged at the downstream end of the injection slot, but did not participate in the later disturbance development. As observed in many experiments $[15,16,17,18]$, the young hairpin vortex developed multiple hairpin vortex heads. A pair of U-shaped vortices developed beneath the hairpin vortex legs. The rotation of these vortices was such that the flow was downward 
near the flow centerline. These vortices were similar to the laterally-displaced secondary vortices reported in the flow visualization experiments of Haidari and Smith [19]; however, the formation of these vortices seemed to be closely connected to the initial injection and not representative of a generic process of vortex formation. The strong dependence of these additional vortices upon the initial conditions suggests that this case might be better classified as a multiple hairpin vortex generation from a single pulse, rather than the single hairpin vortex generation that was the focus of the studies of Haidari and Smith [19].

Haidari and Smith [19] and Smith, Walker, Haidari, and Sobrun [14] describe what they call subsidiary vortices in the flow. These vortices develop from kinks in the vorticity lines that extend through the hairpin vortices. The fact that the formation mechanism of subsidiary vortices is inviscid suggests that the process might be important at high Reynolds numbers, even though we found no significant subsidiary vortices in our simulation. Some waviness in the vorticity lines that go through the hairpin vortices was observed, but this waviness did not lead to the formation of new vortices. Although we did not see any subsidiary vortices, at later times in the computation, laterallydisplaced secondary vortices did develop that were entirely consistent with the Haidari and Smith [19] observations.

Vortices in the flow helped to spawn new vortices. One regenerative process is associated with the ejection of fluid due to an unsteady separation 
process. The ejection is consistent with the high-Reynolds number theory of Van Dommelen and Cowley [12] and the calculations of Peridier, Smith, and Walker $[10,11]$. The theory shows that low-vorticity fluid in a region of adverse pressure gradient will become squeezed in the direction of flow and will be violently ejected from the boundary layer. New vortices can form in the shear layers on either side of the ejection. The theory [12] and calculations $[10,11]$ employed Lagrangian coordinates in order to resolve the very sharply focused events that occur at high Reynolds number. We performed our direct numerical simulation with Eulerian coordinates. Because of the relatively low Reynolds number of the computation, the sharp focusing of events was not as intense and our calculation procedure was able to capture the details. In the direct numerical simulation data presented here, we saw the unsteady separation process primarily in crossflow planes. The process created quasistreamwise vortices in the wall region of the flow. The development of some of the hairpin vortex heads could be attributed to a similar mechanism acting in streamwise-vertical planes, but the uplift of fluid away from the wall is these cases was more gradual, and much of the low-vorticity fluid that was compressed and ejected was already far from the wall before it was exposed to the adverse pressure gradient. Other vortices formed under conditions that were dissimilar to those required by the Van Dommelen and Cowley [12] separation theory. Factors such as the viscous entrainment of fluid by nearby vortices and the rebounding of fluid from the wall played roles in 
the formation of some vortices. In this calculation, many quasi-streamwise vortices were formed near the wall; transverse vortices appeared further from the wall. This activity is consistent with the vortex population observations of Robinson, Kline, and Spalart [5] and Robinson [7] in numerically simulated low Reynolds number, turbulent boundary layers.

Although the flow became increasingly complex, some common patterns were observed. Many quasi-streamwise vortices first appeared under the elongated streamwise legs of other vortices. High-pressure zones often appeared between these vortices. Secondary hairpin vortex heads developed from fluid that had already been lifted from the near-wall region.

The assortment of vortices created an arrowhead-shaped disturbance area in the flow. Both vertical vorticity and streamwise velocity perturbations indicated similar shapes. In profile, the highly intermittent disturbance region had both an overhang portion in the front and a long, low region in the rear. The shape of this region resembled that of a turbulent spot. No waves were detected near the wing tips of the disturbed area. Within the disturbed region, locally averaged, streamwise velocity profiles had a small logarithmic region, but these profiles were not typical of a fully developed turbulent boundary layer. 


\section{Acknowledgments}

The authors would like to thank Dr. Craig Streett and Dr. Thomas Zang for many useful suggestions. The work of Dr. Gokhan Danabasoglu on the original version of the spatial simulation code is also gratefully acknowledged. The authors appreciate the clever suggestions of Dr. David Banks with regard to the graphical presentation of some data. The Theoretical Flow Physics Branch at NASA Langley Research Center supported the first author under contract no. NAS1-20059. Computing resources were provided by NASA's Numerical Aerodynamic Simulation computing facility. 


\section{References}

[1] T. Theodorsen, "Mechanism of Turbulence," Proc. 2nd Midwestern Conf. Fluid Mech., Columbus, Ohio, 1 (1952).

[2] M. R. Head and P. Bandyopadhyay, "New Aspects of Turbulent Boundary-Layer Structure," J. Fluid Mech. 107, 297 (1981).

[3] A. E. Perry, T. T. Lim, and E. W. Teh, “A Visual Study of Turbulent Spots," J. Fluid Mech. 104, 387 (1981).

[4] N. D. Sandham and L. Kleiser, "Vortex Formation in the Late Stages of Transition to Turbulence," Royal Aero. Soc. Boundary Layer Transition and Control Conf., Cambridge, U.K., 26.1 (1991).

[5] S. K. Robinson, S. J. Kline, and P. R. Spalart, "A Review of QuasiCoherent Structures in a Numerically Simulated Turbulent Boundary Layer," NASA TM-102191 (1989).

[6] S. K. Robinson, "A Review of Vortex Structures and Associated Coherent Motions in Turbulent Boundary Layers," Proceedings of Second IUTAM Symposium on Structure of Turbulence and Drag Reduction, Zurich, (1989).

[7] S. K. Robinson, "The Kinematics of Turbulent Boundary Layer Structure," NASA TM-103859 (1991). 
[8] S. K. Robinson, "Coherent Motions in the Turbulent Boundary Layer," in Annu. Rev. Fluid Mech. 23, 601 (1991).

[9] M. Asai and M. Nishioka, "Development of Wall Turbulence in Blasius Flow," in Laminar-Turbulent Transition (eds. D. Arnal and R. Michel), Proceedings of the IUTAM Symposium in Toulouse, France, 1989, Springer-Verlag, 215 (1990).

[10] V. J. Peridier, F. T. Smith, and J. D. A. Walker, "Vortex-induced boundary-layer separation. Part 1 . The unsteady limit problem $R e \rightarrow$ œ," J. Fluid Mech. 232, 99 (1991).

[11] V. J. Peridier, F. T. Smith, and J. D. A. Walker, "Vortex-induced boundary-layer separation. Part 2. Unsteady interacting boundary-layer theory," J. Fluid Mech. 232, 133 (1991).

[12] L. L. van Dommelen and S. J. Cowley, "On the Lagrangian description of unsteady boundary layer separation. Part 1. General theory," J. Fluid Mech. 210, 593 (1990).

[13] S. J. Cowley, L. L. van Dommelen, and S. T. Lam, "On the use of lagrangian variables in descriptions of unsteady boundary-layer separation," Phil. Trans. R. Soc. Lond. A 333, 343 (1990). 
[14] C. R. Smith, J. D. A. Walker, A. H. Haidari, and U. Sobrun, "On the Dynamics of Near-Wall Turbulence," Phil. Trans. R. Soc. Lond. A 336, 131 (1991).

[15] M. S. Acarlar and C. R. Smith, "A Study of Hairpin Vortices in a Laminar Boundary Layer. Part 1. Hairpin Vortices Generated by a Hemisphere Protuberance," J. Fluid Mech. 175, 1 (1987).

[16] M. S. Acarlar and C. R. Smith, "A Study of Hairpin Vortices in a Laminar Boundary Layer. Part 2. Hairpin Vortices Generated by Fluid Injection," J. Fluid Mech. 175, 42 (1987).

[17] H. A. Haidari, B. Taylor, and C. Smith, "The Generation and Growth of Single Hairpin Vortices," AIAA Paper No. 89-0964, (1989).

[18] H. A. Haidari, "Generation and Growth of Single Hairpin Vortices," Ph.D. Dissertation, Mech. Eng. Dept., Lehigh University, Bethlehem, PA, (1990).

[19] H. A. Haidari and C. R. Smith, "The Generation and Regeneration of Single Hairpin Vortices," Accepted for publication J. Fluid Mech. (1994).

[20] J. Amini and G. Lespinard, "Experimental Study of an 'Incipient Spot' in a Transitional Boundary Layer," Phys. Fluids 25, 1743 (1982). 
[21] B. A. Singer, "The Formation and Growth of a Hairpin Vortex," in Instability, Transition, and Turbulence (eds. M. Y. Hussaini, A. Kumar, and C. L. Streett), 367 (1992).

[22] D. S. Henningson, A. Lundbladh, and A. V. Johansson, "A Mechanism for Bypass Transition from Localized Disturbances in Wall Bounded Shear Flows," J. Fluid Mech. 250, 169 (1993).

[23] R. D. Joslin, C. L. Streett, and C.-L. Chang, "Validation of ThreeDimensional Incompressible Spatial Direct Numerical Simulation Code: A Comparison with Linear Stability and Parabolic Stability Theories for Boundary-Layer Transition on a Flat Plate," NASA TP-3205 (1992).

[24] R. D. Joslin, C. L. Streett, and C.-L. Chang, "Spatial Direct Numerical Simulation of Boundary-Layer Transition Mechanisms: Validation of PSE Theory," Theoret. Comput. Fluid Dyn. 4, 271 (1993).

[25] G. Danabasoglu, S. Biringen, and C. L. Streett, "Spatial Simulation of Instability Control by Periodic Suction Blowing," Phys. Fluids A 3, 2138 (1991).

[26] T. A. Zang and M. Y. Hussaini, "Numerical Experiments on the Stability of Controlled Boundary Layers," ICASE Rep. 88-20 (1988).

[27] Z. Shi, J. M. Wu, and J. Z. Wu, "Symmetric and Asymmetric Jets in a Uniform Crossflow," AIAA Paper No. 91-0722 (1991). 
[28] J. J. Greco, "The Flow Structure in the Vicinity of a Cylinder-Flat Plate Junction: Flow Regimes, Periodicity, and Vortex Interactions," MS Thesis, Dept. of ME/Mech., Lehigh University, Bethleham, PA, (1990).

[29] J. P. Hagen and M. Kurosaka, "Corewise Cross-flow Transport in Hairpin Vortices-the 'Tornado Effect'," Phys. Fluids A 5, 3167 (1993).

[30] D. Coles and S. J. Barker, "Some Remarks on a Synthetic Turbulent Boundary Layer," In Turbulent Mixing in Nonreactive and Reactive Flows (ed. S. N. B. Murthy), Plenum, 285 (1975).

[31] I. Wygnanski, M. Sokolov, and D. Friedman, "On a Turbulent 'Spot' in a Laminar Boundary Layer," J. Fluid Mech. 78, 785, (1976).

[32] I. Wygnanski, M. Zilberman, and J. Haritonidis, "On the Spreading of a Turbulent Spot in the Absence of a Pressure Gradient," J. Fluid Mech. 123, 69 (1982).

[33] J. Barrow, F. H. Barnes, M. A. S. Ross, and S. T. Hayes, "The Structure of a Turbulent Spot in a Blasius Flow," J. Fluid Mech. 149, 319 (1984).

[34] A. Glezer, Y. Katz, and I. Wygnanski, "On the Breakdown of the Wave Packet Trailing a Turbulent Spot in a Laminar Boundary Layer," J. Fluid Mech. 198, 1 (1989). 
[35] Y. Katz, A. Seifert, and I. Wygnanski, "On the Evolution of the Turbulent Spot in a Laminar Boundary Layer with a Favourable Pressure Gradient," J. Fluid Mech. 221, 1 (1990).

[36] I. Wygnanski, J. H. Haritonidis, and R. E. Kaplan, "On a TollmienSchlichting Wave Packet Produced by a Turbulent Spot," J. Fluid Mech. 92, $505(1979)$.

[37] F. W. Chambers and A. S. W. Thomas, "Turbulent Spots, Wave Packets, and Growth," Phys. Fluids 261160 (1983).

[38] D. Henningson, P. Spalart, and J. Kim, "Numerical Simulations of Turbulent Spots in Plane Poiseuille and Boundary-Layer Flow," Phys. Fluids 30, 2914 (1987).

[39] D. S. Henningson and J. Kim, “On Turbulent Spots in Plane Poiseuille Flow," J. Fluid Mech. 228, 183 (1991). 


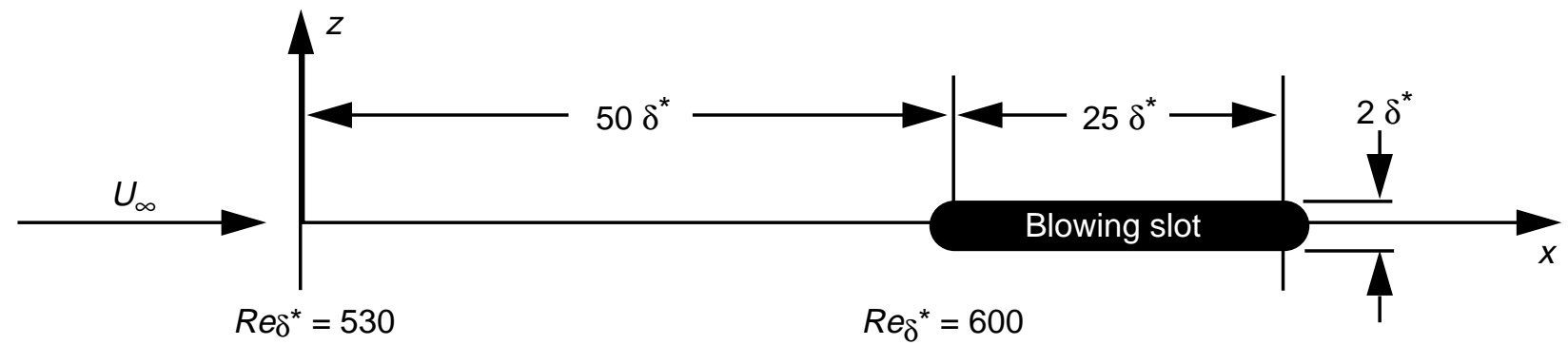

(a)
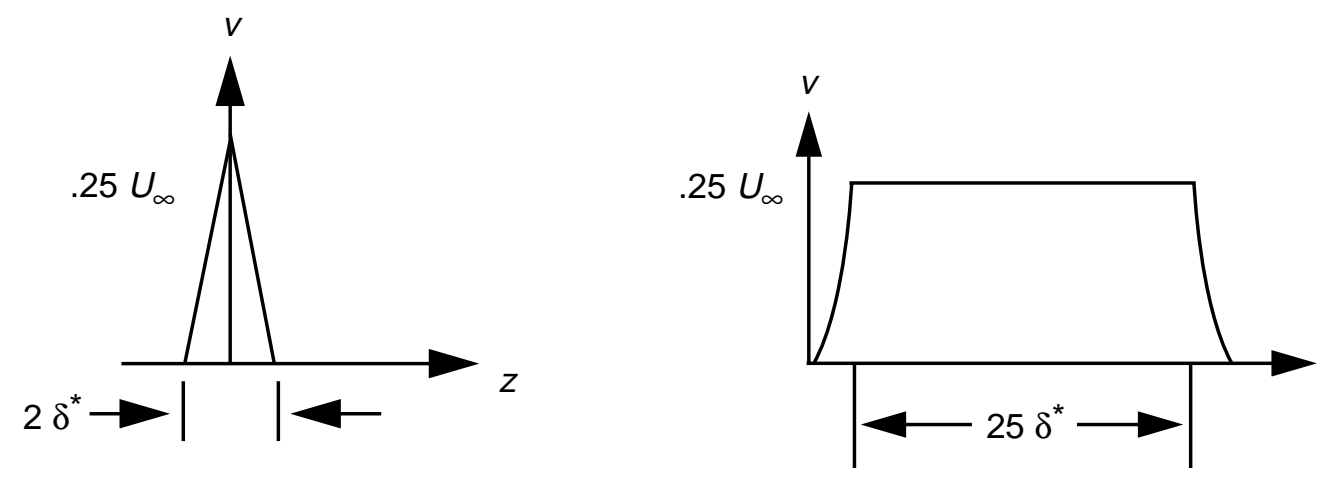

(b)

Fig. 1. Details of localized injection. Reynolds numbers are based on local displacement thickness. Lengths are nondimensionalized on displacement thickness at $R e_{\delta^{*}}=530$. (a) Schematic of computational domain and injection slot. Solid black region along symmetry line shows location of injection slot. (b) Vertical velocity distribution along symmetry line during times of maximum injection $(0.5 \leq t \leq 4.5)$. View on lower left illustrates spanwise distribution of vertical velocity. 


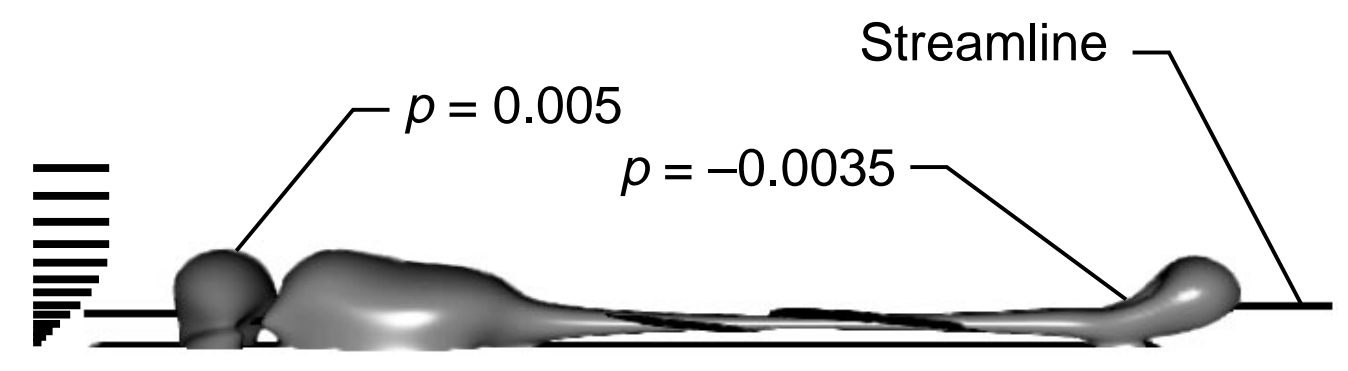

Fig. 2. Side view of isopressure surfaces at $t=15.0$. Total velocity vectors on centerline upstream of injection slot are illustrated on left. Thin black horizontal line represents region over which fluid injection occurred. Streamline that starts just upstream of injection region spirals around streamwise elongated low-pressure region. 


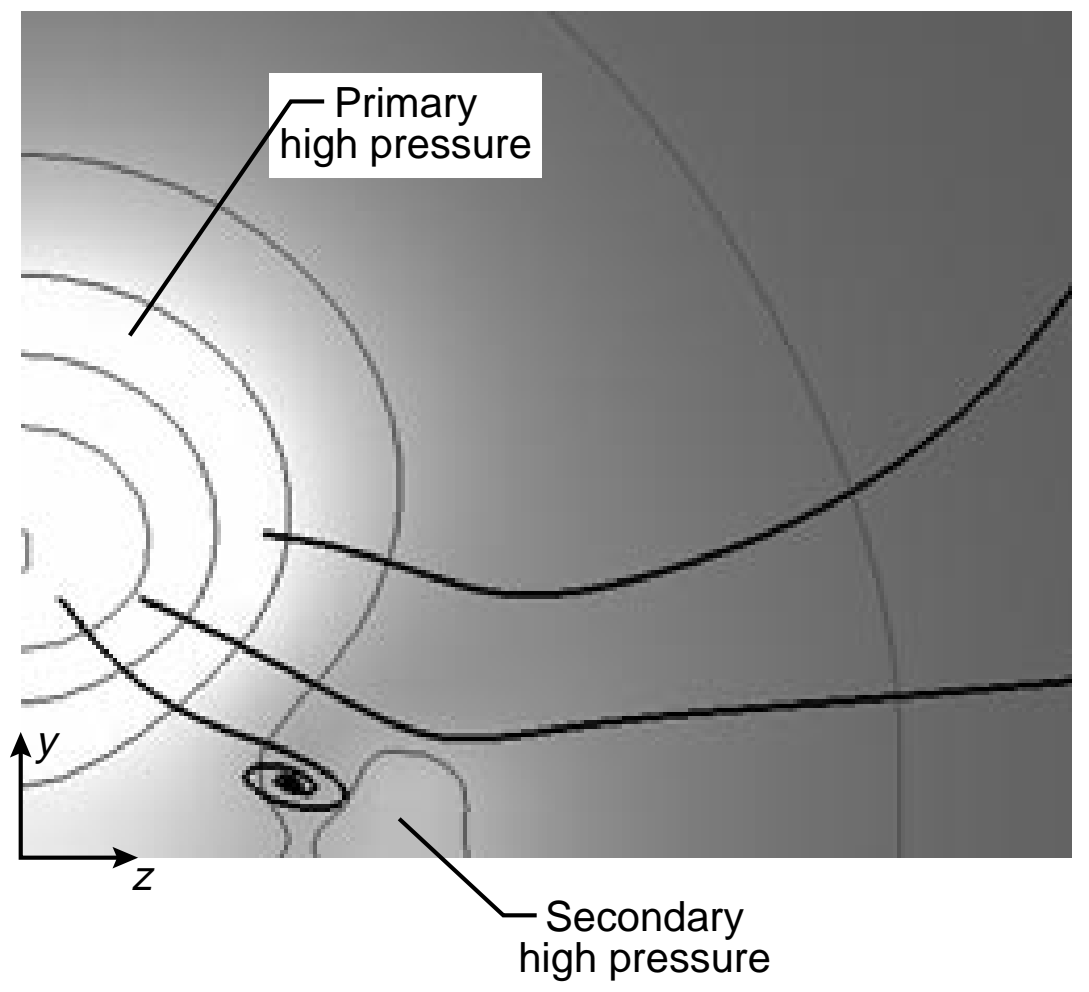

Fig. 3. Grey-scale pressure contours at $t=21.75$ in plane $x=55.1$. Light areas are high pressure; dark areas are low pressure. Grey contour lines are superimposed. Streamlines restricted to $x=55.1$ plane are shown as black lines. Only $z>0$ portion of plane is shown. 


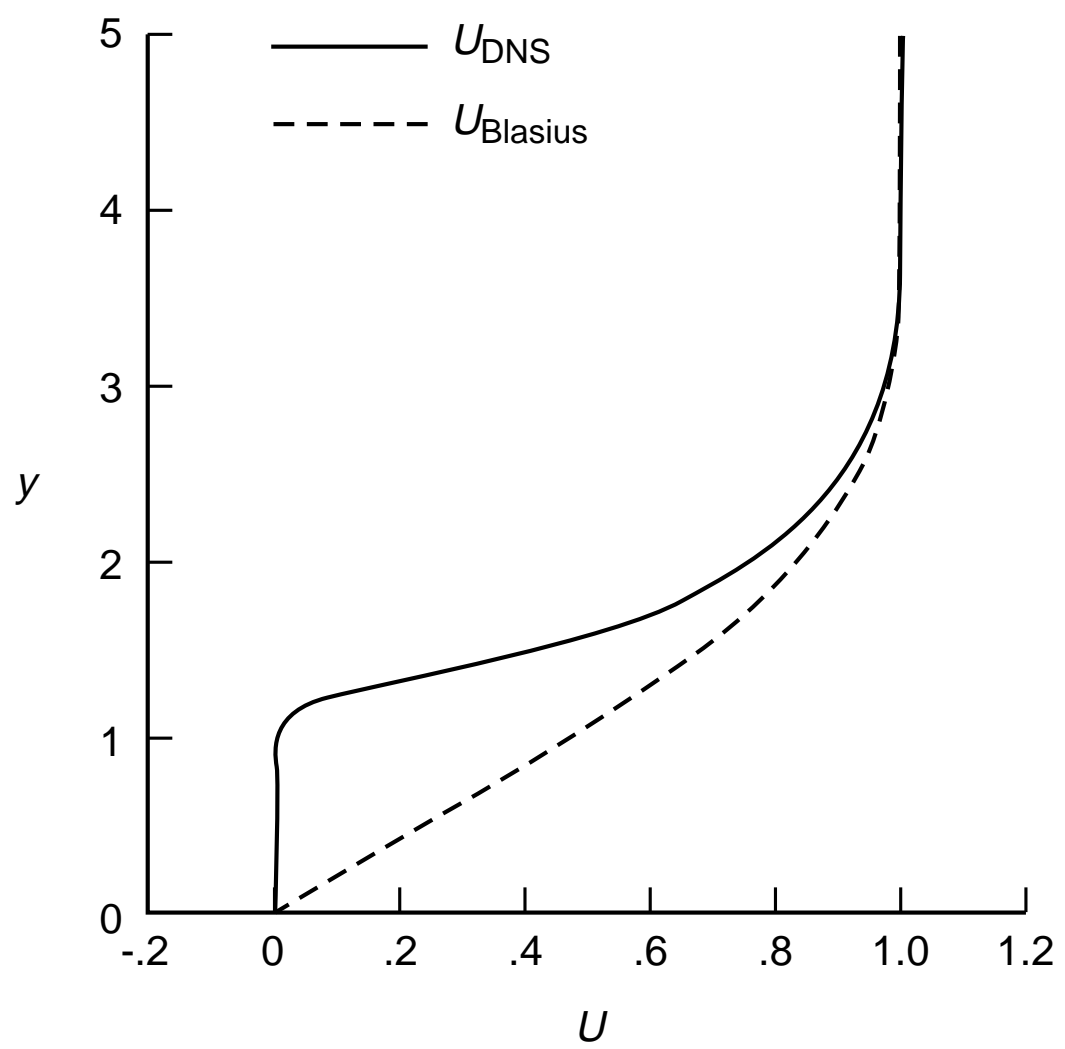

Fig. 4. Streamwise velocity profile at $x=65.2$ and $t=15.0$. Blasius profile at same location is included for comparison. 


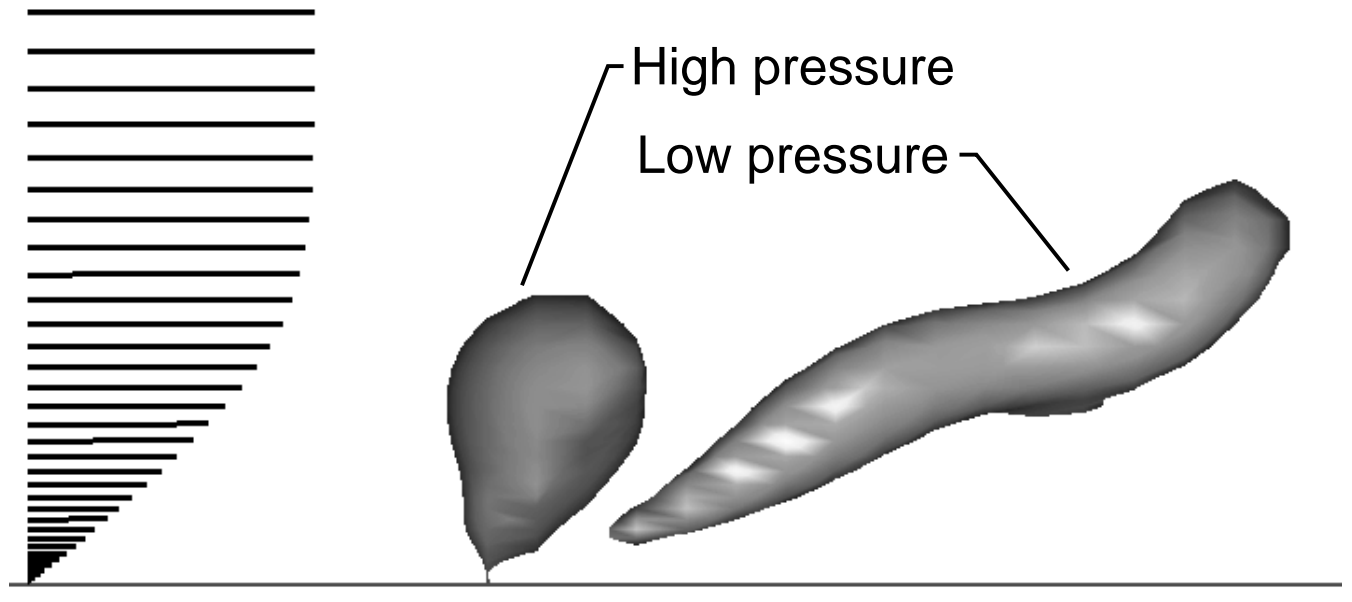

Fig. 5. Side view of high- and low-pressure regions $(p= \pm 0.035)$ at $t=35.70$. Low-pressure region resembles a hairpin vortex. Blasius velocity profile is shown on left. Downstream end of hairpin vortex is at $x=65.7$; velocity profile is at $x=56.9$. 


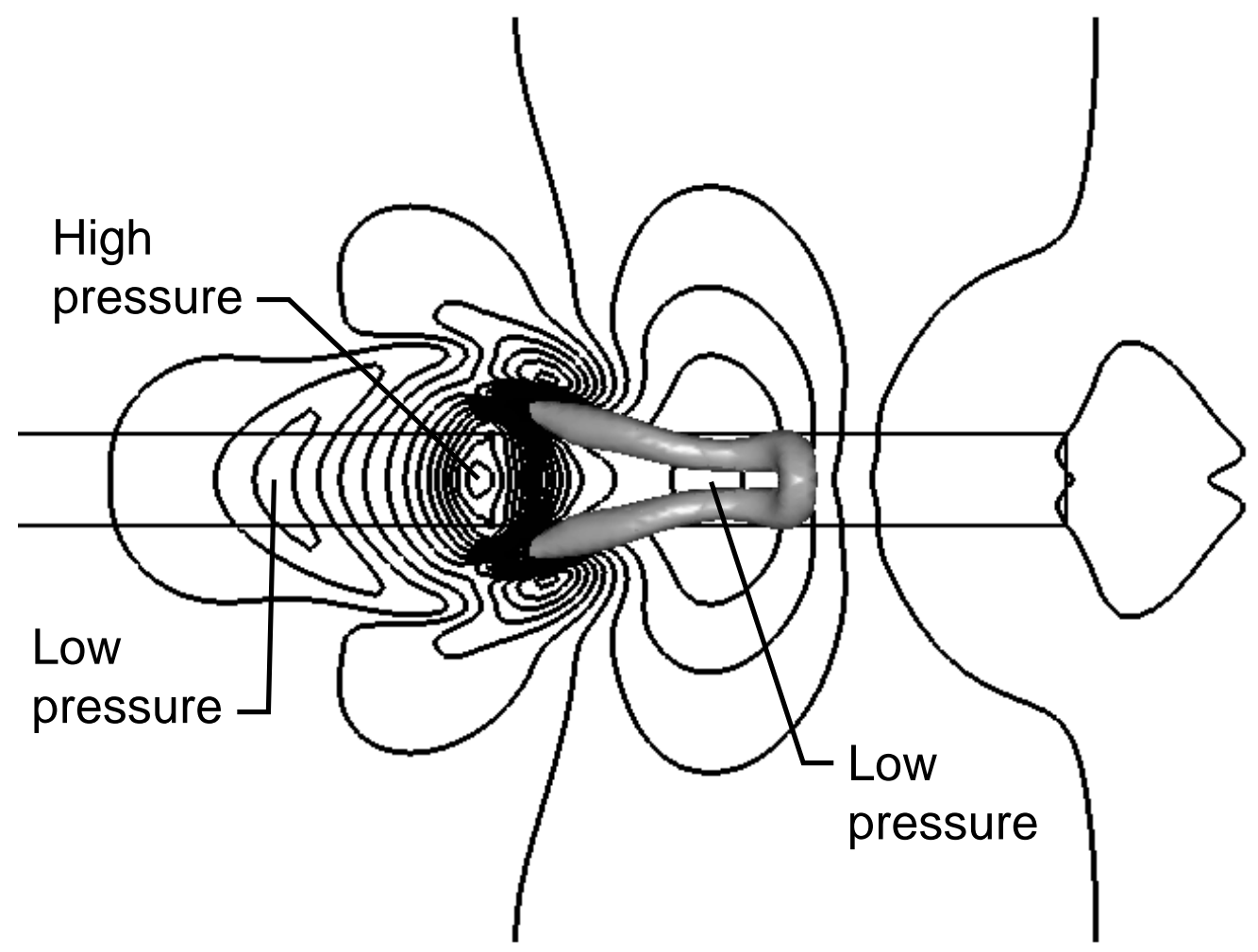

Fig. 6. Plan view of pressure contours at $t=42.0$ in plane $y=0.53$. Lightly colored three-dimensional surface represents hairpin vortex structure. Rectangle (which is open on left) is downstream end of injection slot. Core of necklace vortex is approximately represented by U-shaped low-pressure contours upstream of hairpin vortex. 


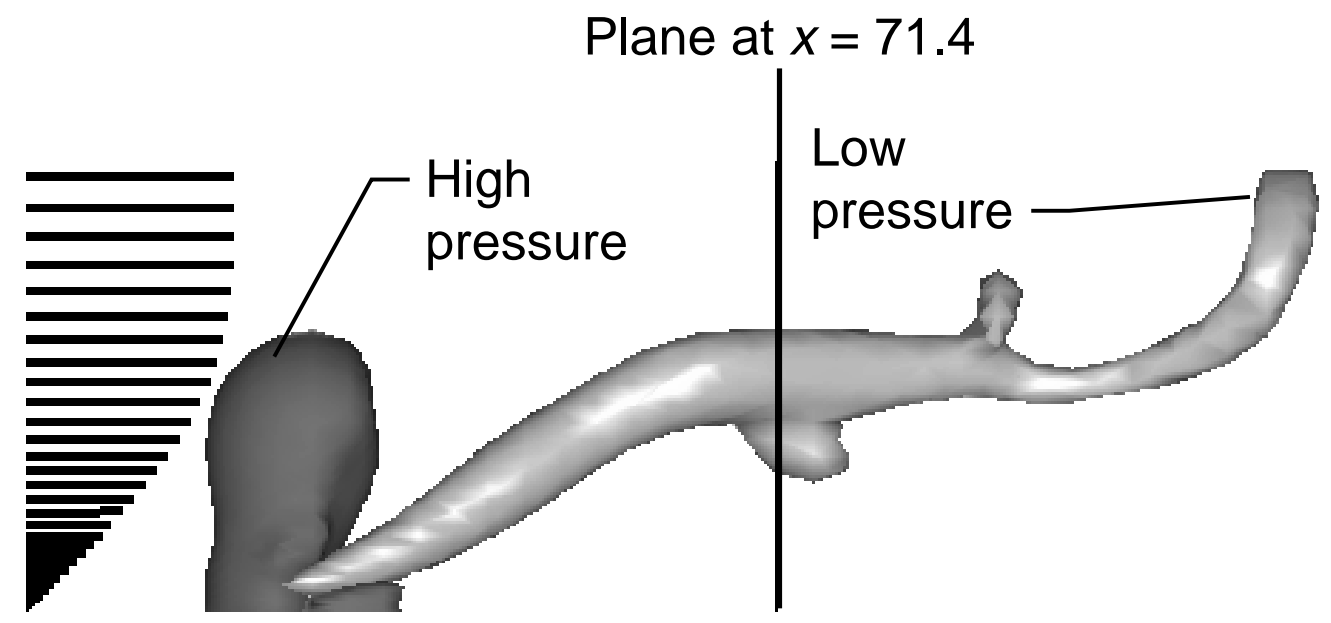

Fig. 7. Side view of high- and low-pressure regions $(p= \pm 0.035)$ at $t=50.25$. Blasius velocity profile is shown on left. Low-pressure zones show primary hairpin vortex, secondary vortex head, and U-shaped vortex forming underneath each vortex leg. Vertical line is at $x=71.4$. (See Fig. 9.) Downstream end of hairpin vortex is at $x=76.5$; velocity profile is at $x=64.3$. 


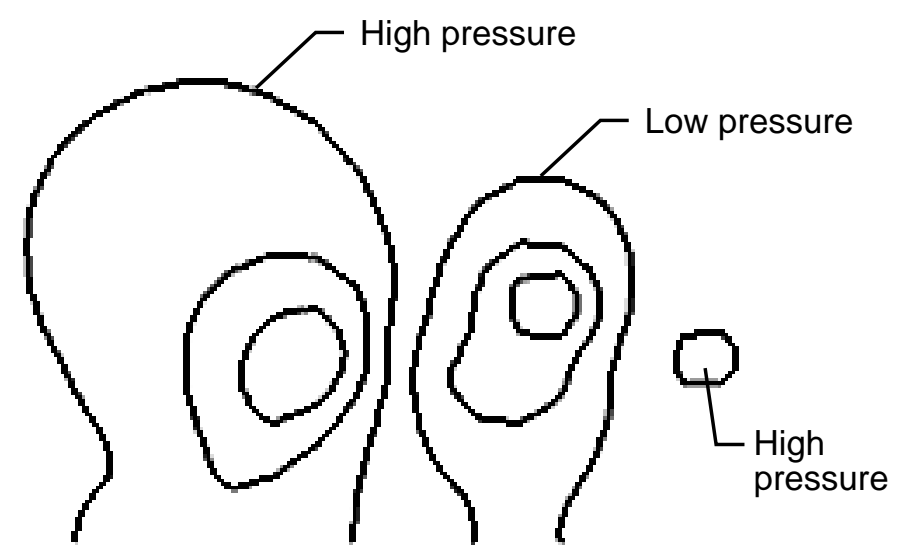

(a)

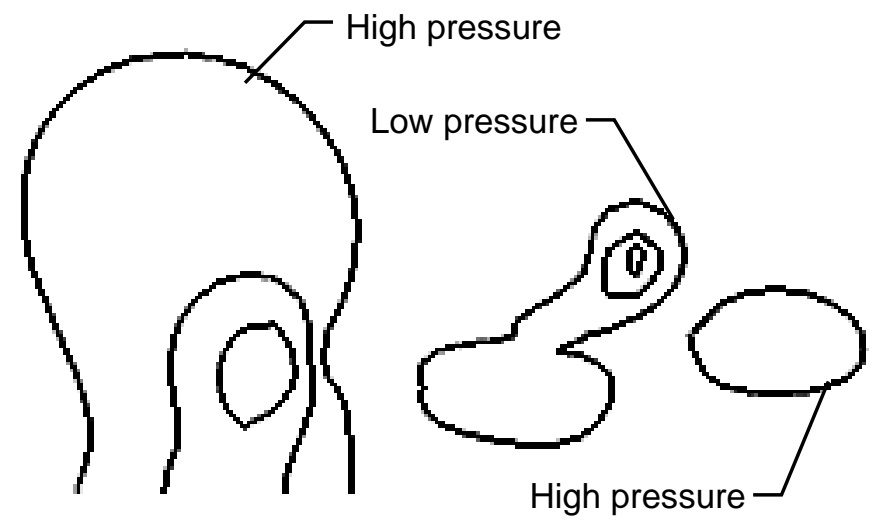

(b)

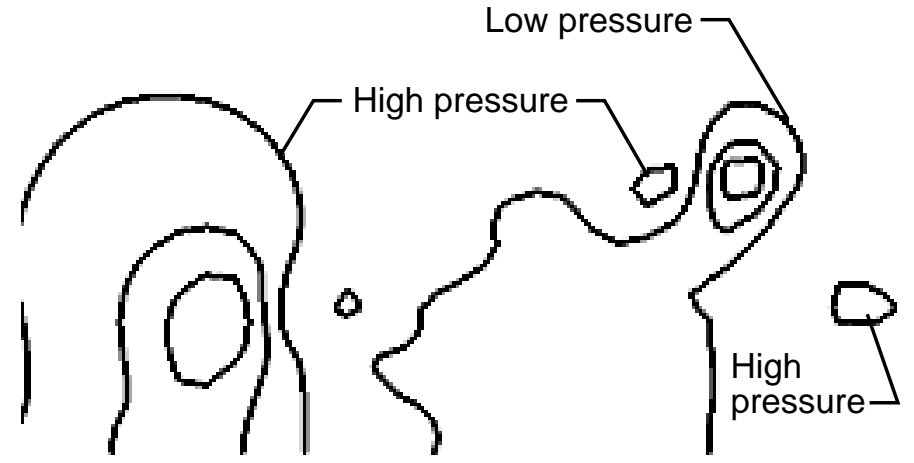

(c)

Fig. 8. Pressure contours on centerline. (a) $t=26.5$. (b) $t=35.7$. (c) $t=42.0$. 

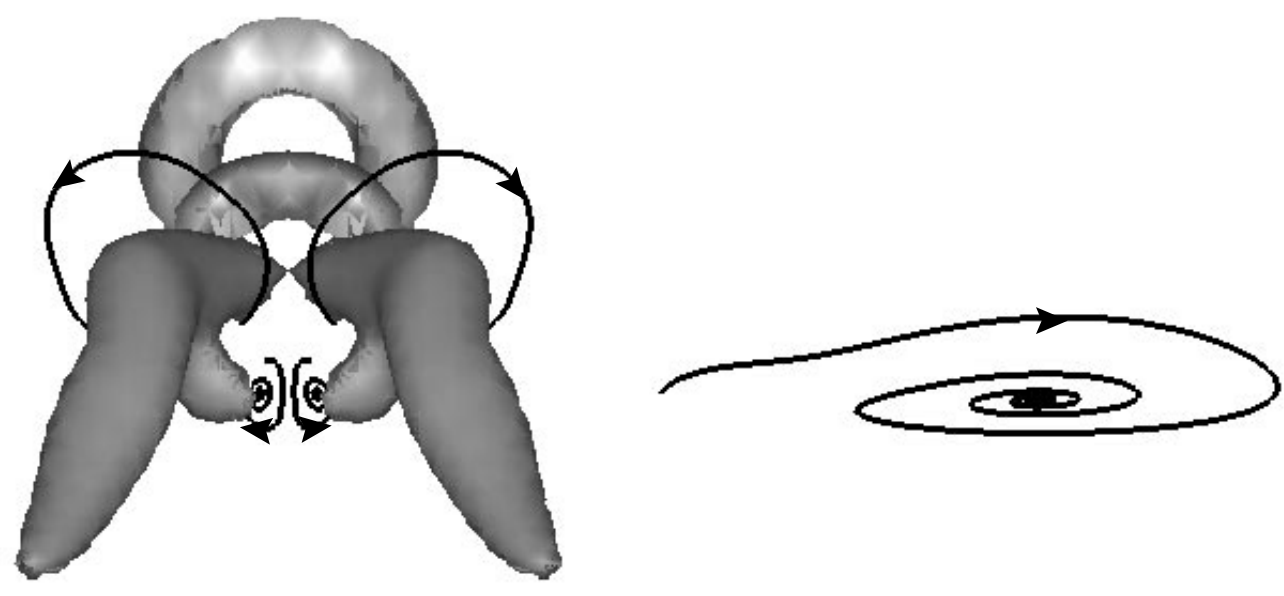

Fig. 9. Downstream view of low-pressure surfaces $(p=-0.036)$ at $t=50.25$. Black lines are streamlines restricted to plane $x=71.4$. (See vertical line of Fig. 7 .) 


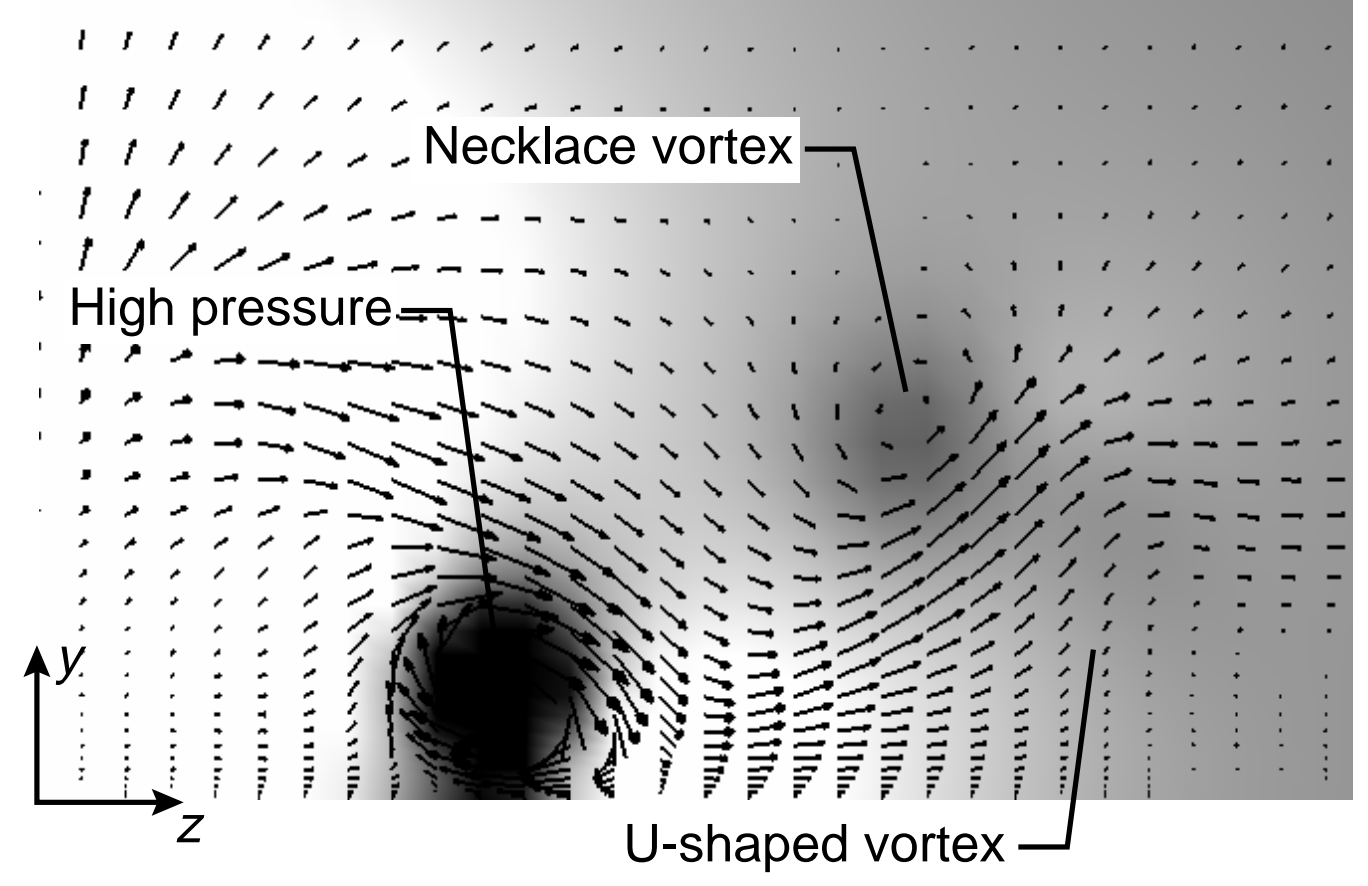

Fig. 10. Grey-scale pressure surfaces on plane $x=67.3$ at $t=50.25$. Perturbation velocity vectors are superimposed. Only $z>0$ portion of plane is shown. 

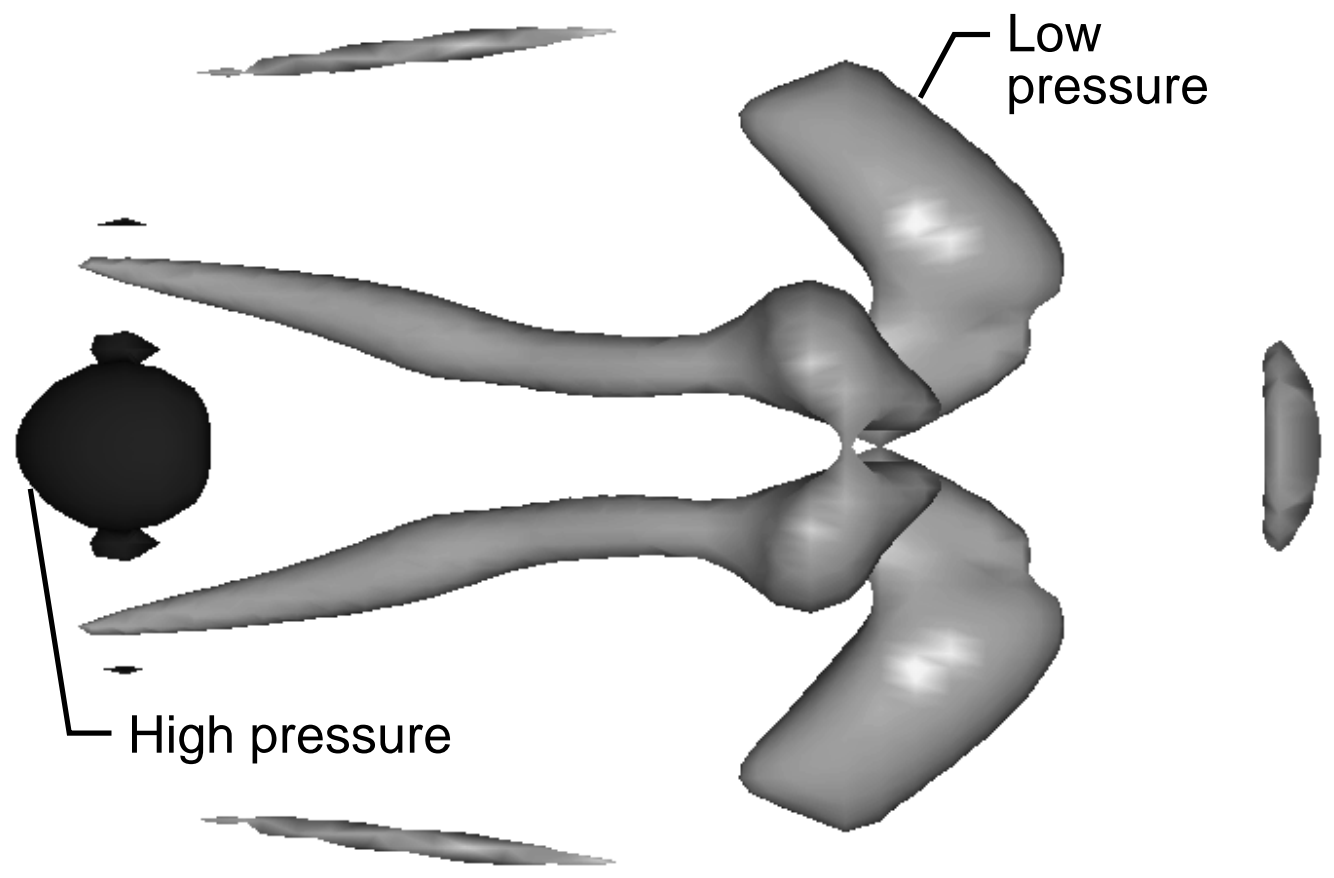

Fig. 11. Plan view of high- $(p=0.03)$ and low-pressure $(p=-0.035)$ regions at $t=80.70$. Downstream edge of vortex head is at $x=94.0$. Upstream end of high-pressure region is at $x=80.5$. 

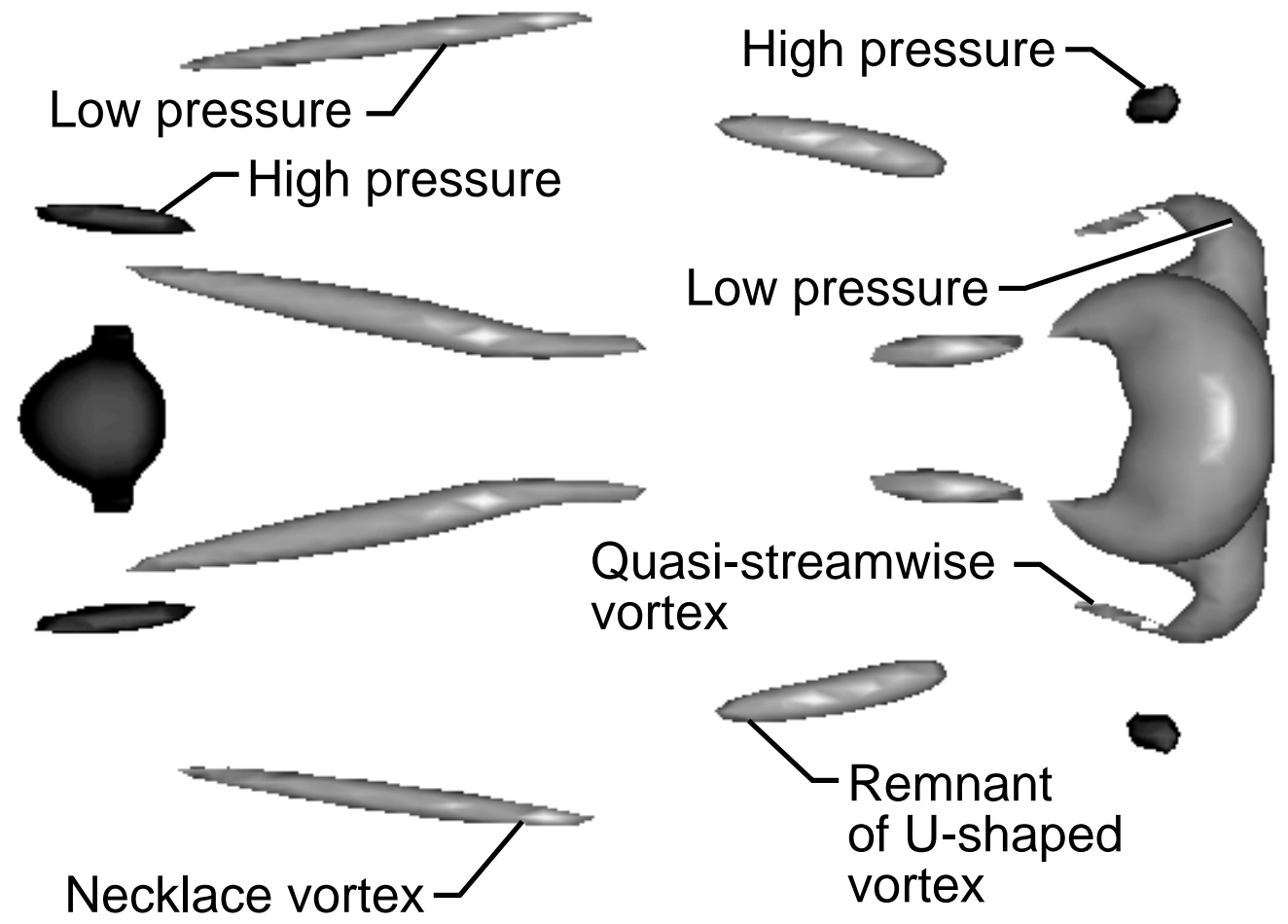

Fig. 12. Plan view of high- $(p=0.02)$ and low-pressure $(p=-0.035)$ regions at $t=97.2$. Downstream edge of vortex head is at $x=103.6$. Upstream end of high-pressure region is at $x=88.6$. 


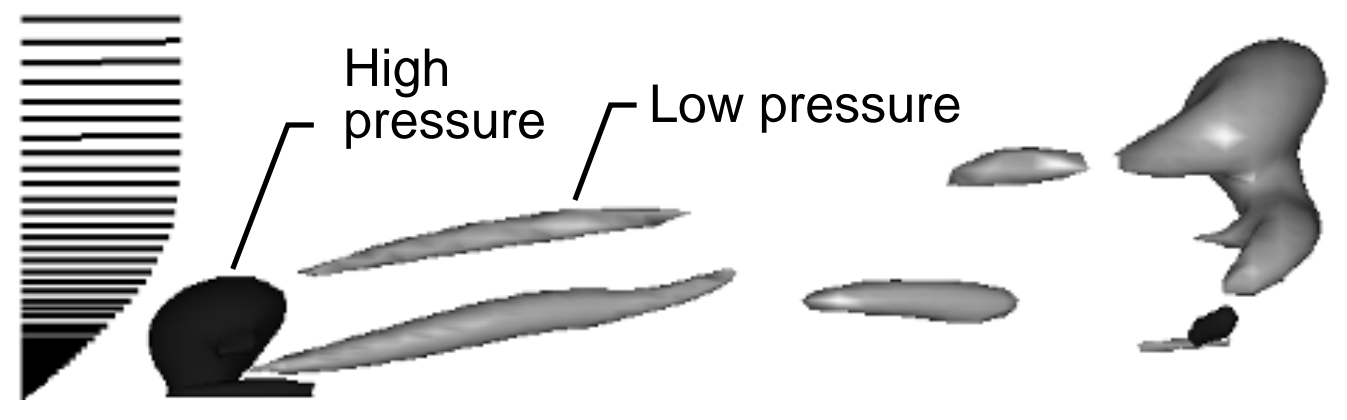

Fig. 13. Side view of high- $(p=0.02)$ and low-pressure $(p=-0.035)$ regions at $t=97.2$. Blasius profile is shown at left at $x=86.9$. 


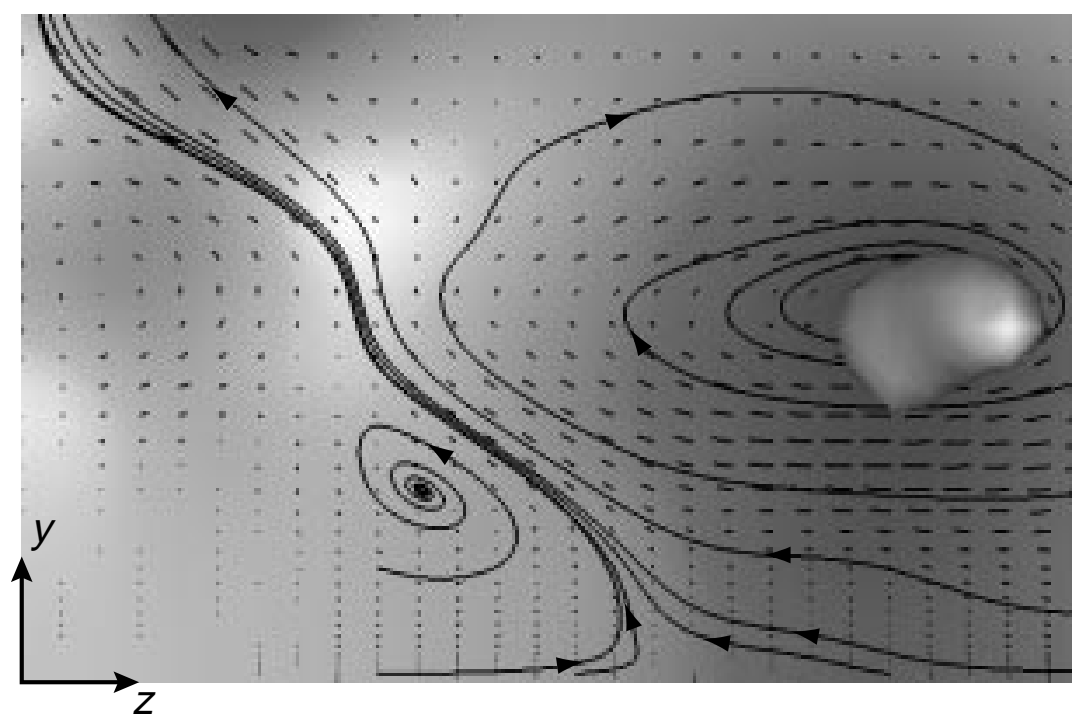

Fig. 14. Cross-sectional plane at $x=98.7$ at $t=97.2$. Light areas are high pressure; dark areas are low pressure. Grey surface on right is portion of remnant of U-shaped vortex that protrudes upstream of plane $x=98.7$. Velocity field is indicated by vector lines. Streamlines restricted to plane are shown. 


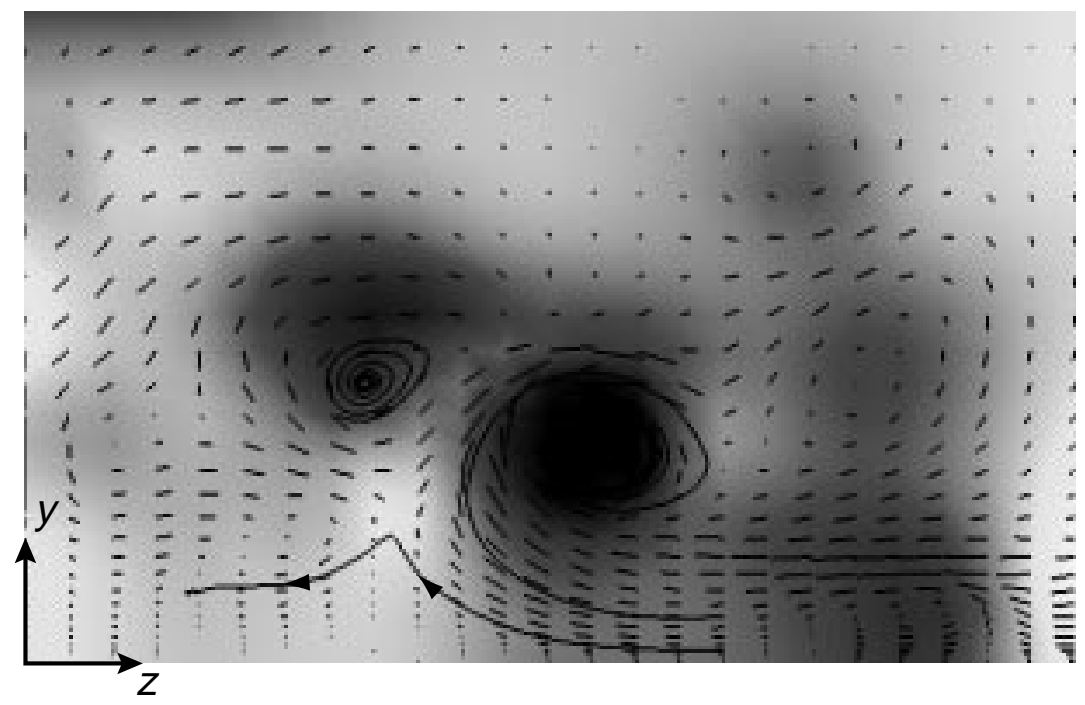

(a)

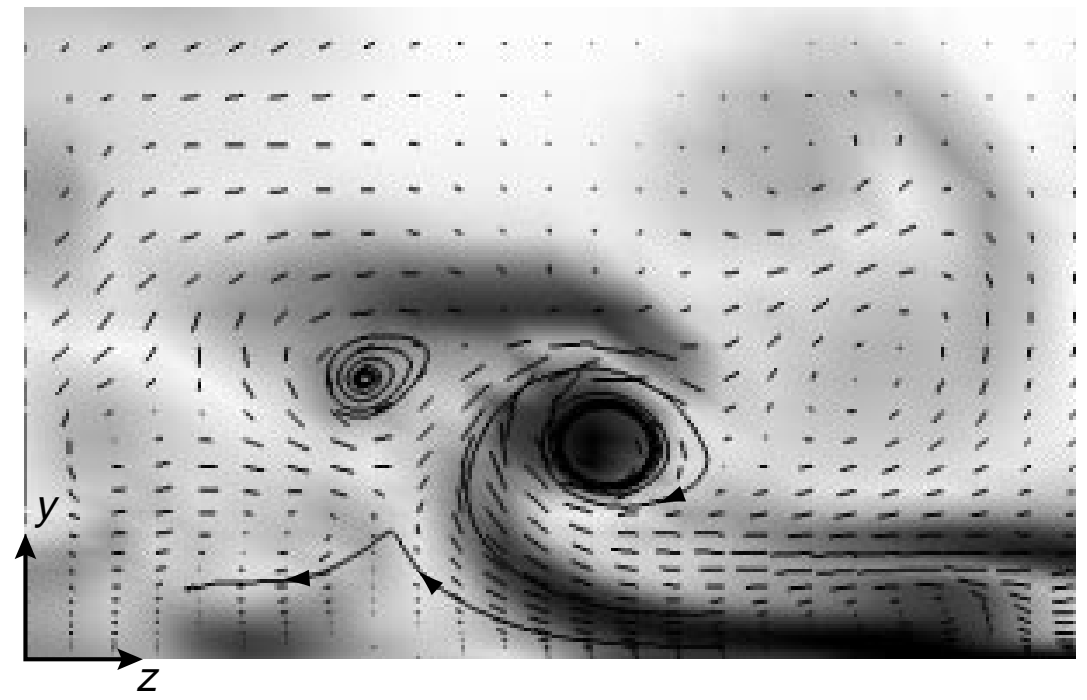

(b)

Fig. 15. Cross-sectional plane $x=128.2$ at time $t=148.0$. Cross-stream velocity vectors and plane-restricted streamlines are shown. (a) Pressure (high in light regions and low in dark regions). (b) Magnitude of vorticity (high in dark regions and low in light regions). 


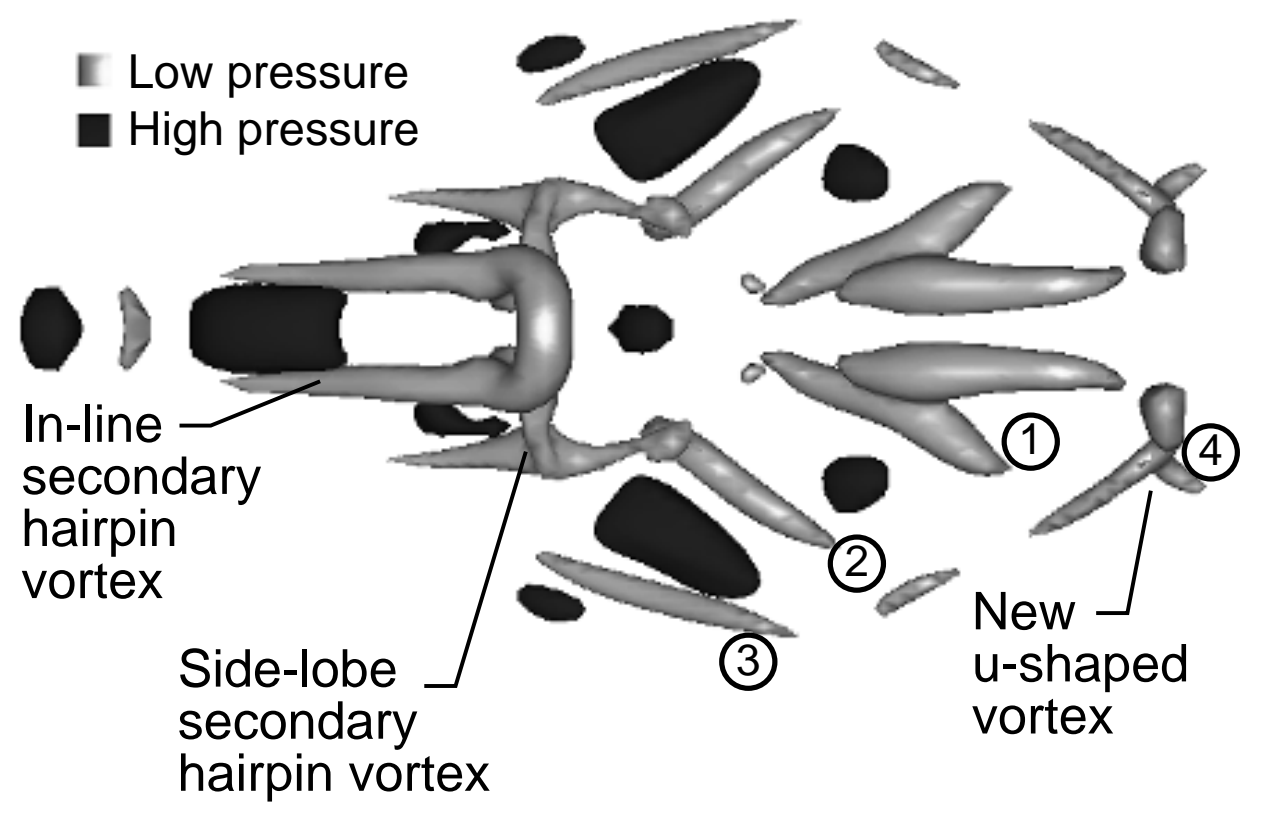

Fig. 16. Plan view of high- $(p=0.02)$ and low-pressure $(p=-0.035)$ regions at $t=153.10$. Downstream edge of vortex is at $x=147.7$. Upstream end of high-pressure region is at $x=120.2$. Numbers indicate order of appearance of quasi-streamwise vortices. 

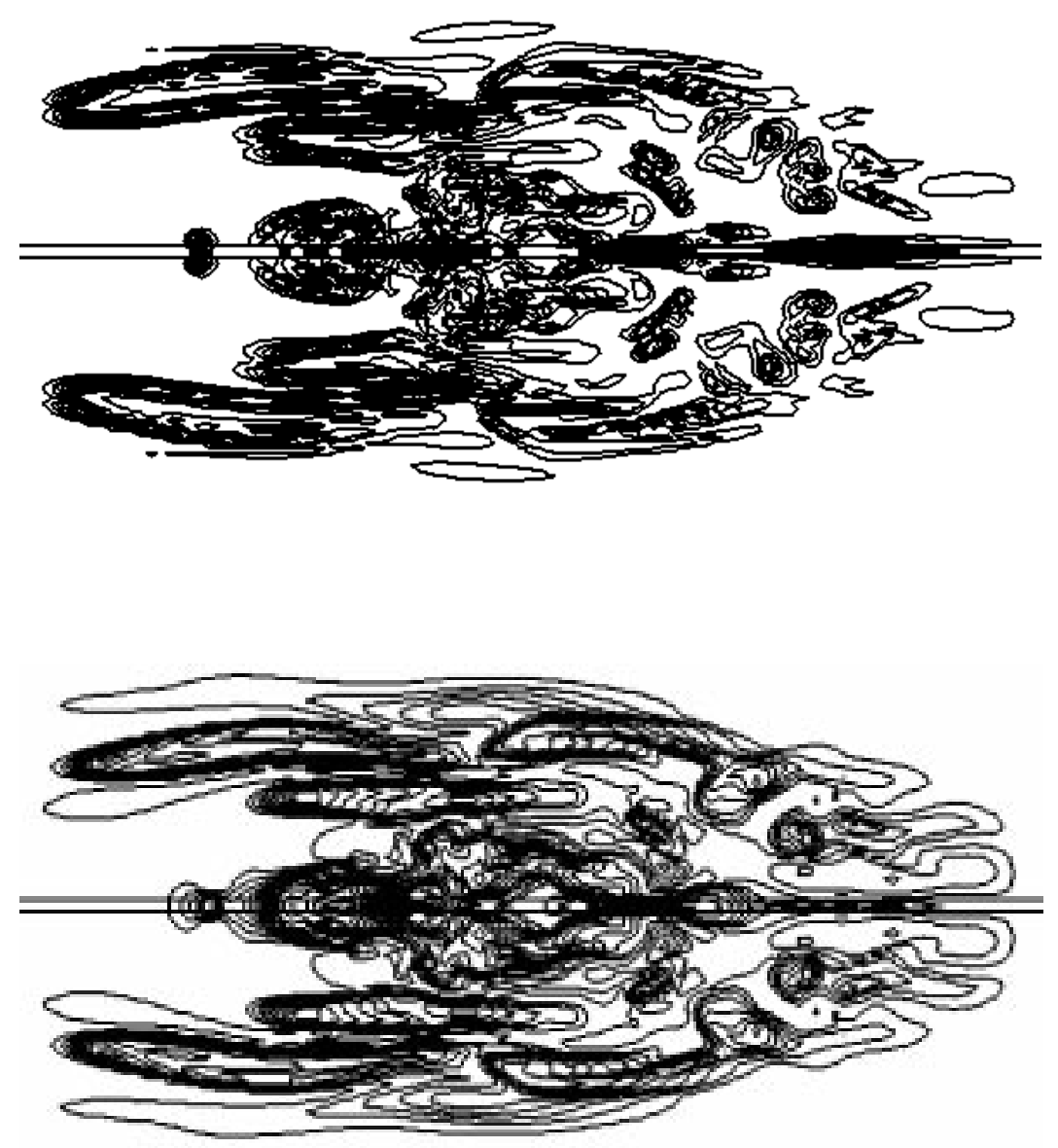

Fig. 17. Plan view of magnitude of perturbation streamwise velocity (bottom) and vertical vorticity (top) at $t=150.10$ and $y=2.41$. Enveloping contours are $0.02 U_{0}$ and $0.02 U_{0} / \delta_{0}^{*}$, respectively. Contour increments are $0.05 U_{0}$ for velocity and $0.10 U_{0} / \delta_{0}^{*}$ for vorticity. Horizontal lines indicate locations where $z= \pm 0.25$ (i.e., locations for side views in following figure). 

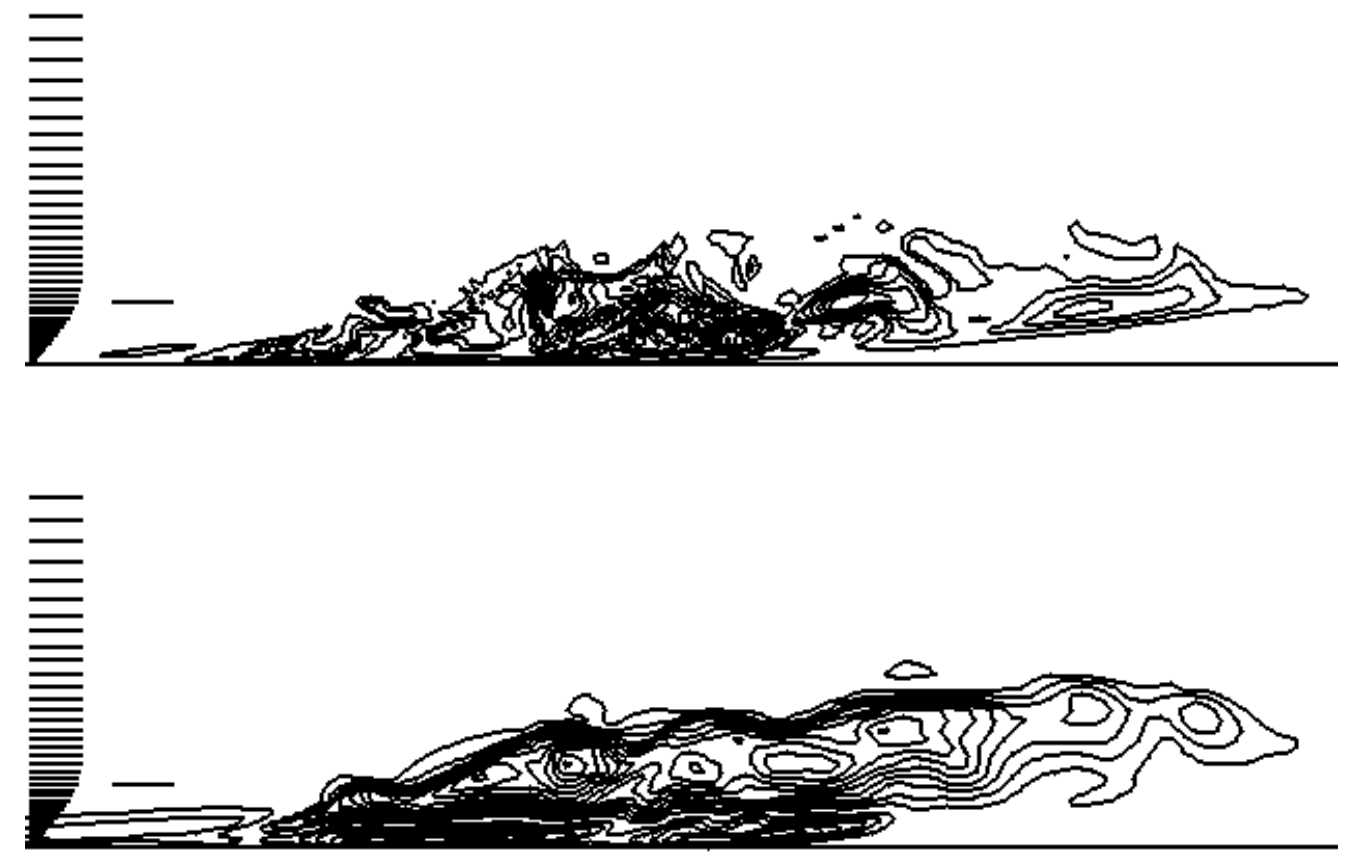

Fig. 18. Side view of magnitude of perturbation streamwise velocity (bottom) and vertical vorticity (top) along $z=0.25$ at $t=150.10$. Enveloping contours are $0.02 U_{0}$ and $0.02 U_{0} / \delta_{0}^{*}$, respectively. Contour increments are $0.05 U_{0}$ for velocity and $0.10 U_{0} / \delta_{0}^{*}$ for vorticity. Horizontal lines on bottoms indicate wall locations. Velocity vectors appear at left. Horizontal line extending beyond velocity vectors indicates position where $y=2.41$ (i.e. location for plan views in previous figures). 


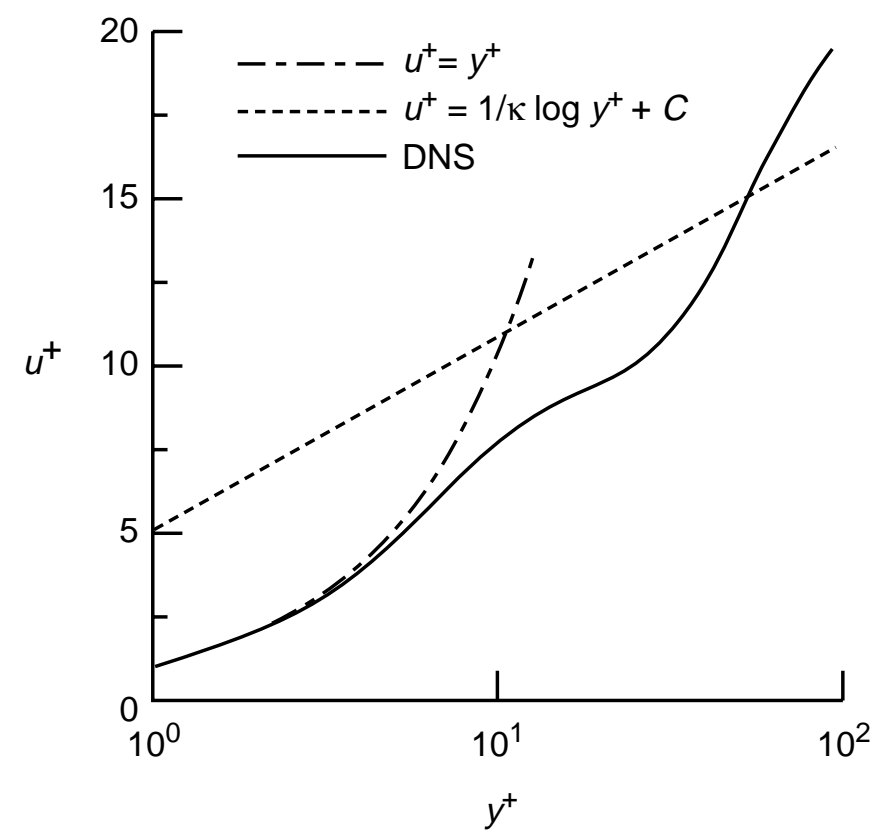

Fig. 19. Locally averaged $(120 \leq x \leq 132,|z| \leq 3.0)$ profile in wall units at $t=158.30$. 